\title{
High-mass Star Formation through Filamentary Collapse and Clump-fed Accretion in G22
}

\author{
Jinghua Yuan ${ }^{1}$ (D), Jin-Zeng $\mathrm{Li}^{1}$, Yuefang $\mathrm{Wu}^{2}$ (D), Simon P. Ellingsen ${ }^{3}$ (D), Christian Henkel ${ }^{4,5}$ (D), Ke Wang ${ }^{6}$ (D), Tie Liu ${ }^{7,8}$ (D), \\ Hong-Li Liu ${ }^{9}$ (D), Annie Zavagno ${ }^{10}$, Zhiyuan Ren ${ }^{1}$, and Ya-Fang Huang ${ }^{1}$ \\ ${ }^{1}$ National Astronomical Observatories, Chinese Academy of Sciences, 20A Datun Road, Chaoyang District, Beijing 100012, China; jhyuan@nao.cas.cn \\ ${ }_{2}^{2}$ Department of Astronomy, Peking University, 100871 Beijing, China \\ ${ }^{3}$ School of Physical Sciences, University of Tasmania, Hobart, Tasmania, Australia \\ ${ }^{4}$ Max-Planck-Institut für, Radioastronomie, Auf dem Hügel 69, D-53121 Bonn, Germany
${ }^{4}$ Astronomy Department, Faculty of Science, King Abdulaziz University, P.O. Box 80203, 21589 Jeddah, Saudi Arabia \\ ${ }_{7}^{6}$ European Southern Observatory, Karl-Schwarzschild-Str. 2, D-85748 Garching bei München, Germany \\ ${ }^{7}$ Korea Astronomy and Space Science Institute 776, Daedeokdae-ro, Yuseong-gu, Daejeon 34055, Korea \\ ${ }^{8}$ East Asian Observatory, 660 N. A'ohōkū Place, Hilo, HI 96720-2700, USA \\ ${ }_{9}^{9}$ Department of Physics, The Chinese University of Hong Kong, Shatin, NT, SAR, Hong Kong \\ ${ }^{10}$ Aix Marseille Univ, CNRS, LAM, Laboratoire d'Astrophysique de Marseille, Marseille, France \\ Received 2017 July 23; revised 2017 November 17; accepted 2017 November 23; published 2017 December 28
}

\begin{abstract}
How mass is accumulated from cloud-scale down to individual stars is a key open question in understanding highmass star formation. Here, we present the mass accumulation process in a hub-filament cloud G22 that is composed of four supercritical filaments. Velocity gradients detected along three filaments indicate that they are collapsing with a total mass infall rate of about $440 M_{\odot} \mathrm{Myr}^{-1}$, suggesting the hub mass would be doubled in six free-fall times, adding up to $\sim 2$ Myr. A fraction of the masses in the central clumps $\mathrm{C} 1$ and $\mathrm{C} 2$ can be accounted for through large-scale filamentary collapse. Ubiquitous blue profiles in $\mathrm{HCO}^{+}(3-2)$ and ${ }^{13} \mathrm{CO}(3-2)$ spectra suggest a clump-scale collapse scenario in the most massive and densest clump $\mathrm{C} 1$. The estimated infall velocity and mass infall rate are $0.31 \mathrm{~km} \mathrm{~s}^{-1}$ and $7.2 \times 10^{-4} M_{\odot} \mathrm{yr}^{-1}$, respectively. In clump C1, a hot molecular core (SMA1) is revealed by the Submillimeter Array observations and an outflow-driving high-mass protostar is located at the center of SMA1. The mass of the protostar is estimated to be 11-15 $M_{\odot}$ and it is still growing with an accretion rate of $7 \times 10^{-5} M_{\odot} \mathrm{yr}^{-1}$. The coexistent infall in filaments, clump C1, and the central hot core in G22 suggests that pre-assembled mass reservoirs (i.e., high-mass starless cores) may not be required to form high-mass stars. In the course of high-mass star formation, the central protostar, the core, and the clump can simultaneously grow in mass via core-fed/disk accretion, clump-fed accretion, and filamentary/cloud collapse.
\end{abstract}

Key words: ISM: clouds - ISM: individual objects (G22) - ISM: kinematics and dynamics - stars: formation stars: massive

\section{Introduction}

The process of mass accumulation in high-mass star formation has remained elusive for decades (Tan et al. 2014; Motte et al. 2017). In the turbulent core model, which is one of the two extensively debated scenarios, the final stellar mass is pre-assembled in the collapsing high-mass core (McKee \& Tan 2003). Otherwise, the competitive accretion model allows the mass reservoir, often referred to as the star-forming core, to keep growing in the course of high-mass star formation (Bonnell et al. 2001).

Prevalent filaments detected in our Galaxy are wellestablished as some of the main sites for star formation (André et al. 2010, 2014; Molinari et al. 2010a; Wang et al. 2014, 2015, 2016). Specifically, hub-filament systems are frequently reported forming high-mass stars (e.g., Hennemann et al. 2012; Liu et al. 2012, 2016; Peretto et al. 2013). Converging flows detected in several hub-filament systems channel gas to the junctions where star formation is often most active (e.g., Kirk et al. 2013; Peretto et al. 2014; Liu et al. 2016). However, how gas flows help individual star-forming cores grow in mass is still poorly understood.

High-mass clumps $(0.3-1 \mathrm{pc})$ are the objects that fragment into dense cores $(<0.3 \mathrm{pc})$, which subsequently contract to form individual or bound systems of protostars. Clump-scale global collapse has been reported in case studies (Schneider et al. 2010; Csengeri et al. 2011; Ren et al. 2012; Liu et al. 2013; Peretto et al. 2013), based on which "clump-fed accretion" has been proposed as an alternative high-mass star formation mode (Motte et al. 2017; Tigé et al. 2017). It still remains unknown, how significant such clump-scale collapse may be in sustaining mass growth in individual cores where high-mass protostars have already formed.

Located in the inner Galactic Plane with $l=22^{\circ} .04$ and $b=0^{\circ} .2$, the G22 cloud contains 10 Spitzer infrared dark clouds (IRDCs) from Peretto \& Fuller (2009). These IRDCs are mainly distributed in a hub-filament system. Several young stellar objects (Robitaille et al. 2008), an extended green object (EGO G022.04+00.22, Cyganowski et al. 2008), and methanol maser emission (Cyganowski et al. 2009) detected in G22 indicate active high-mass star formation therein. These features make G22 an ideal laboratory to investigate how mass is accumulated in the high-mass star formation process, from cloud-scale down to individual cores. Using the Bayesian Distance Calculator developed by Reid et al. (2016), the distance to G22 is estimated to be $3.51 \pm 0.28 \mathrm{kpc}$ based on its systemic velocity of $50.9 \mathrm{~km} \mathrm{~s}^{-1}$.

In this work, we carry out an extensive study of G22 based on observations with varying angular resolutions to reveal a promising high-mass star formation scenario where the central 
high-mass protostar, the core, and the clump grow in mass simultaneously. The data used in this work are described in Section 2. We present results about the large-scale cloud and the Submillimeter Array (SMA) observations in Sections 3 and 4. More in-depth discussions and a summary of the findings are given in Sections 5 and 6.

\section{Data}

\subsection{Galactic Plane Survey Data}

We extracted multi-wavelength data covering the entire cloud from the Galactic Legacy Infrared Mid-Plane Survey Extraordinaire (GLIMPSE), MIPSGAL, Hi-GAL and ATLASGAL surveys. Using the IRAC instrument on board the Spitzer Space Telescope (Werner et al. 2004), the GLIMPSE (Benjamin et al. 2003; Churchwell et al. 2009) project surveyed the inner $130^{\circ}$ of the Galactic Plane at 3.6, 4.5, 5.8, and $8.0 \mu \mathrm{m}$ with $5 \sigma$ sensitivities of $0.2,0.2,0.4$, and $0.4 \mathrm{mJy}$, respectively. The Galactic Plane Survey using the Multiband Infrared Photometer on Spitzer (MIPSGAL; Cyganowski et al. 2009) mapped an area comparable to GLIMPSE at longer infrared wavelengths. Version 3.0 of the MIPSGAL data provides mosaics from only the $24 \mu \mathrm{m}$ band with an angular resolution of $6^{\prime \prime}$ and a $5 \sigma$ sensitivity of about $1.7 \mathrm{mJy}$. As a key project of the Herschel Space Observatory, the Herschel Infrared Galactic Plane Survey (Hi-GAL; Molinari et al. 2010b) mapped the entire Galactic plane with nominal $|b|<1^{\circ}$ (following the Galactic warp) at 70, 160, 250, 350, and $500 \mu \mathrm{m}$ with angular resolutions of 10 !" 2,13 !" 5,18 !. 1 , 25 ! 0 , and 36". 4. Using the LABOCA camera, the APEX Telescope Large Areas Survey of the Galaxy (ATLASGAL Schuller et al. 2009) mapped 420 square degrees of the Galactic plane between $-80^{\circ}<l<60^{\circ}$ at $870 \mu \mathrm{m}$ with a 19 !' 2 angular resolution. Cutouts of $8^{\prime} \times 8^{\prime}$ were retrieved from the above surveys to reveal the large-scale morphology and global properties of the entire cloud.

\subsection{Single Dish Molecular Data}

From the FCRAO Galactic Ring Survey (GRS, Jackson et al. 2006) we retrieved ${ }^{13} \mathrm{CO} J=1-0$ line data of the cloud. The GRS ${ }^{13} \mathrm{CO}$ data have velocity and angular resolutions of $0.21 \mathrm{~km} \mathrm{~s}^{-1}$ and $46^{\prime \prime}$. The main beam efficiency $\left(\eta_{\mathrm{mb}}\right)$ and the typical rms sensitivity are 0.48 and $0.13 \mathrm{~K}$, respectively.

From the JCMT data archive, we extracted ${ }^{13} \mathrm{CO}(3-2)$, $\mathrm{C}^{18} \mathrm{O}(3-2)$, and $\mathrm{HCO}^{+}(3-2)$ data, which cover only the central $\sim 1^{\prime} \times 1^{\prime}$. The ${ }^{13} \mathrm{CO}(3-2)$ and $\mathrm{C}^{18} \mathrm{O}(3-2)$ were simultaneously observed in 2007 August with an angular resolution of $15^{\prime \prime}$, a velocity resolution of $0.055 \mathrm{~km} \mathrm{~s}^{-1}$, and an rms noise of about $1.20 \mathrm{~K}$. Observed in 2008 May, the $\mathrm{HCO}^{+}(3-2)$ data have an angular resolution of $20^{\prime \prime}$, a velocity resolution of $0.55 \mathrm{~km} \mathrm{~s}^{-1}$, and an rms noise of about $0.18 \mathrm{~K}$.

\subsection{SMA Observations}

Observations toward the central massive clump $\mathrm{C} 1$ using the SMA at $1.3 \mathrm{~mm}$ were performed on 2010 August 10th in the compact-north configuration with eight antennae used. The data are publicly available in the SMA data archive (PI: Claudia Cyganowski). The $2 \times 4 \mathrm{GHz}$ correlator was tuned to cover the frequency range $216.8-220.8 \mathrm{GHz}$ in the lower sideband (LSB) and $218.8-232.8 \mathrm{GHz}$ in the upper sideband (USB). The $4 \mathrm{GHz}$ bandwidth in each sideband is allocated to 48 spectral windows, each of which consists of 128 channels with an uniform width of $812.5 \mathrm{kHz}$ $\left(\sim 1.1 \mathrm{~km} \mathrm{~s}^{-1}\right)$. The projected baseline lengths ranged from 8 to $105 \mathrm{k} \lambda$, which indicates that the SMA observations are insensitive to smoothed structures larger than $20^{\prime \prime}$.

3c454.3, J1733-130/J1911-201, and Callisto (Jupiter's moon) were observed as bandpass, gain, and flux density calibrators. The data were reduced and imaged using MIRIAD (Sault et al. 1995). GILDAS (Pety 2005), APLpy ${ }^{11}$, and Astropy (Astropy Collaboration et al. 2013) packages were used for data visualization and analysis. The continuum data were constructed using line free channels from both sidebands, which resulted in a synthesized beam of 2 ". $95 \times 1$ " 92 $\left(\mathrm{PA}=68^{\circ} .4\right)$ and an rms noise of $3.2 \mathrm{mJy}^{\text {beam }^{-1}}$. For the line data, the continuum emission was subtracted by modeling an order one polynomial. We also performed self-calibration to the continuum data and applied the solutions to the line data. The typical noise in a single channel of the line data is about $80 \mathrm{mJy}^{\text {beam }^{-1}}$.

\section{A Hub-filament Cloud}

Cutouts at wavelengths from 3.6 to $870 \mu \mathrm{m}$ of the $\mathrm{G} 22$ cloud are shown in Figure 1. Extinction features, which correspond to 10 infrared dark clouds identified by Peretto \& Fuller (2009), dominate in the north at $8.0 \mu \mathrm{m}$ and can be partly seen at 24 and $70 \mu \mathrm{m}$. Extended emission in the south at 8.0, 24, and 70 $\mu \mathrm{m}$ originates from an infrared dust bubble, MWP1G022027 +002159 (Simpson et al. 2012). This infrared dust bubble is slightly farther from us but physically interacting with G22 (see Section 5.4).

As shown in Figures 1(b)-(h), two clumps (C1 and C2, see bellow) dominate emission at wavelengths longer than $24 \mu \mathrm{m}$. These two clumps also represent the most active star-forming regions in G22. Detected at far-IR and sub-mm wavelengths, several filaments converge at clumps $\mathrm{C} 1$ and $\mathrm{C} 2$.

\subsection{Dust Properties}

Column density and dust temperature maps have been obtained via fitting the spectral energy distribution (SED) to the multi-bands data. To reach an intermediate angular resolution, we only used data at $160,250,350$, and $870 \mu \mathrm{m}$ to perform the fitting. The images were convolved to a common angular resolution of $25^{\prime \prime}$, which is essentially the poorest resolution of the considered wavelengths. The convolution kernels of Aniano et al. (2011) were used to take the different instrumental responding functions into account. For the ATLASGAL images, any uniform astronomical signal on spatial scales larger than 2.5 has been filtered out together with atmospheric emission during the data reduction (Schuller et al. 2009). The filtering of Hi-GAL images was performed using the CUPID-findback algorithm of the Starlink suite. For further details on the algorithm, please see the online document for findback. ${ }^{12}$ Following Yuan et al. (2017), we run the algorithm iteratively using a common filtering box of 2.5 to get stable background images that were subtracted from the postconvolution data for Hi-GAL bands to remove large-scale structures.

\footnotetext{
11 APLpy is an open-source plotting package for Python hosted at http:// aplpy.github.com.

12 http://starlink.eao.hawaii.edu/starlink/findback.html
} 


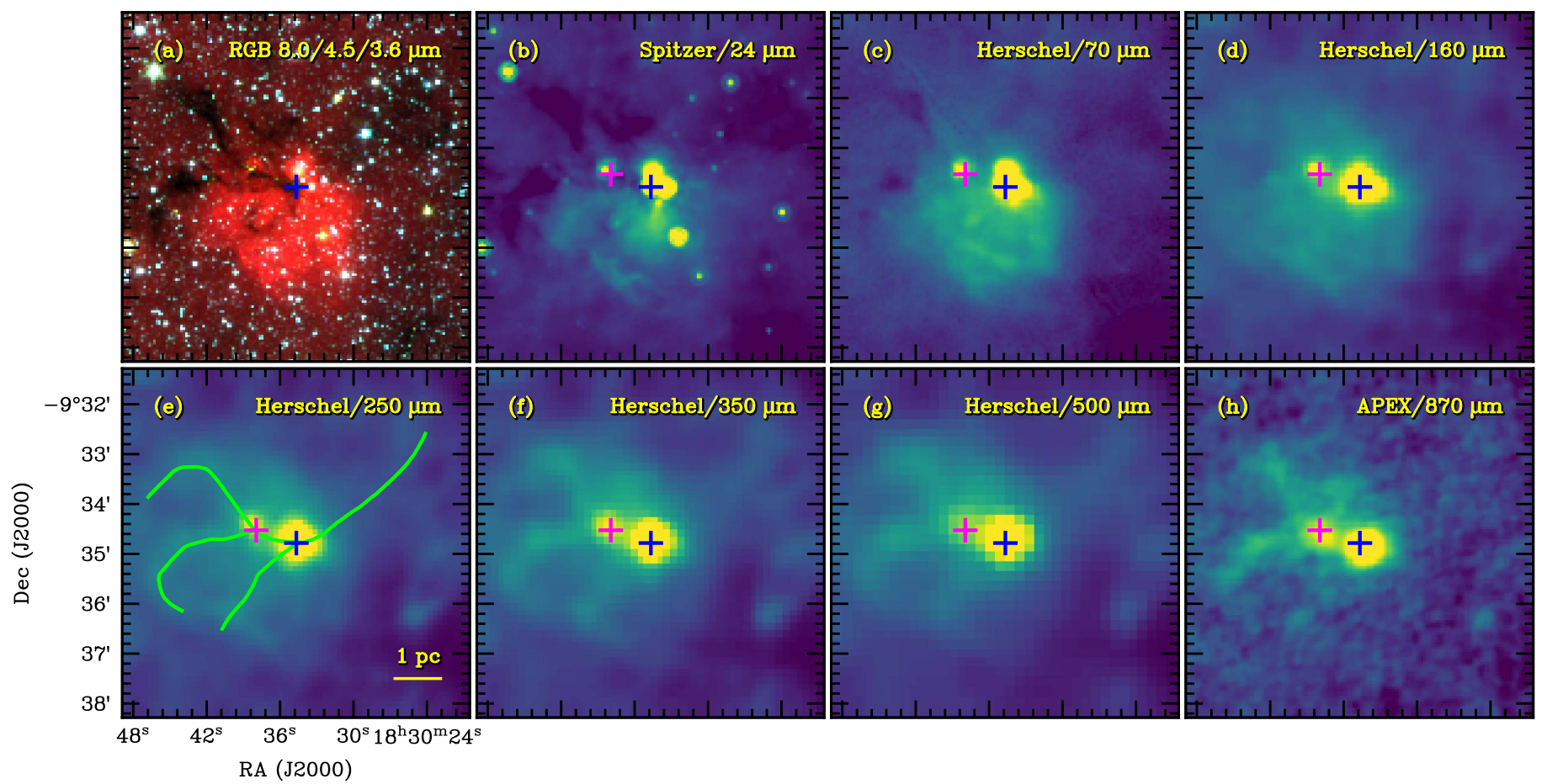

Figure 1. Morphology of G022.04+0.22 in multi-wavelength bands. The blue and magenta crosses mark the most massive clumps C1 and C2 (see Figure 2 and Section 3.1) in the hub region. The skeletons of the four filaments are also shown in (e). The Hi-GAL images shown here are the unfiltered ones.

Intensity as a function of wavelength for each pixel was obtained from the smoothed and background-removed far-IR to sub-mm image data and modeled to a modified blackbody.

$$
I_{\nu}=B_{\nu}(T) \mu_{\mathrm{H}_{2}} m_{\mathrm{H}} \kappa_{\nu} N_{\mathrm{H}_{2}} / R_{\mathrm{gd}}
$$

where

$$
\kappa_{\nu}=\kappa_{600}\left(\frac{\nu}{600 \mathrm{GHz}}\right)^{\beta} \mathrm{cm}^{2} \mathrm{~g}^{-1} .
$$

Here, $\mu_{\mathrm{H}_{2}}=2.8$ is the mean molecular weight adopted from Kauffmann et al. (2008), $m_{\mathrm{H}}$ is the mass of a hydrogen atom, $N_{\mathrm{H}_{2}}$ is the $\mathrm{H}_{2}$ column density, $R_{\mathrm{gd}}=100$ is the gas to dust ratio, $\kappa_{600}=5.0 \mathrm{~cm}^{2} \mathrm{~g}^{-1}$ is the dust opacity at $600 \mathrm{GHz}$ for coagulated grains with thin ice mantles (Ossenkopf \& Henning 1994). The dust emissivity index $\beta$ was fixed to 1.75 (Ossenkopf \& Henning 1994; Planck Collaboration et al. 2014). Free parameters in this model are the dust temperature $N_{\mathrm{H}_{2}}$ and column density $T_{\text {dust }}$.

The resultant column density and dust temperature maps are presented in Figure 2. We note that the dust temperatures will be underestimated, as the modeling only considered emission at wavelengths not shorter than $160 \mu \mathrm{m}$, while some regions significantly emit at $70 \mu \mathrm{m}$. Uncertainties in $N_{\mathrm{H}_{2}}$ and $T_{\text {dust }}$ can also originate from the dust opacity, which is subject to a factor of two uncertainty (Ossenkopf \& Henning 1994). The dust emissivity index $(\beta)$ can also largely influence the resultant parameters. An increase of 0.5 for $\beta$ would lead to a $2 \%-35 \%$ increase for $N_{\mathrm{H}_{2}}$ and a $5 \%-18 \%$ decrease for $T_{\text {dust }}$, and $N_{\mathrm{H}_{2}}$ would decrease by $10 \%-35 \%$ and $T_{\text {dust }}$ might increase by $13 \%-28 \%$ if $\beta$ decreases by 0.5 (Yuan et al. 2017).
From the $N_{\mathrm{H}_{2}}$ map, we have visually identified four filamentary structures, which intersect at the center to constitute a hub-filament system. These four filaments are named as F1 to F4 and listed in Table 1 together with some physical parameters. Their loci are overlaid in Figure 2(a). Nine $N_{\mathrm{H}_{2}}$ peaks were identified as clumps and fitted to two-dimensional (2D) Gaussian profiles using the 2D Fitting Tool of the CASA viewer. Their FK5 coordinates, and major and minor axes $\left(\Theta_{\text {maj }}\right.$ and $\left.\Theta_{\text {min }}\right)$ are given in Table 2. These clumps are designated as $\mathrm{C} 1$ to $\mathrm{C} 9$ and delineated as open ellipses in Figure 2(a). Interestingly, six of the nine clumps are located inside filaments with the most massive one $(\mathrm{C} 1)$ in the intersecting hub.

Clump masses were estimated via integrating the column densities in the Gaussian ellipses. Source-averaged $\mathrm{H}_{2}$ number densities and mass surface densities were calculated assuming a spherical morphology with a constant density profile and an equivalent radius of $r_{\text {eq }}=d \sqrt{\Theta_{\text {maj }} \Theta_{\text {min }}-\theta_{\text {beam }}^{2}} / \sqrt{2 \ln 2}$, where $\theta_{\text {beam }}=25^{\prime \prime}$. The resultant equivalent radii, clump masses, $\mathrm{H}_{2}$ number densities, and source-averaged surface densities are given in Table 2 together with peak $\mathrm{H}_{2}$ column densities and source-averaged dust temperatures. These eight clumps have masses ranging from 62 to $590 M_{\odot}$, radii ranging from 0.30 to $0.67 \mathrm{pc}$. The total mass of all clumps is $2189 M_{\odot}$, holding about $47 \%$ of the cloud mass $\left(5943 M_{\odot}\right)$.

\subsection{Molecular Emission}

The velocity-integrated intensity map of ${ }^{13} \mathrm{CO}(1-0)$ is shown in Figure 3. Although filamentary features are smoothed out due to the poor angular resolution, the strongest emission is still spatially coincident with the hub region. 


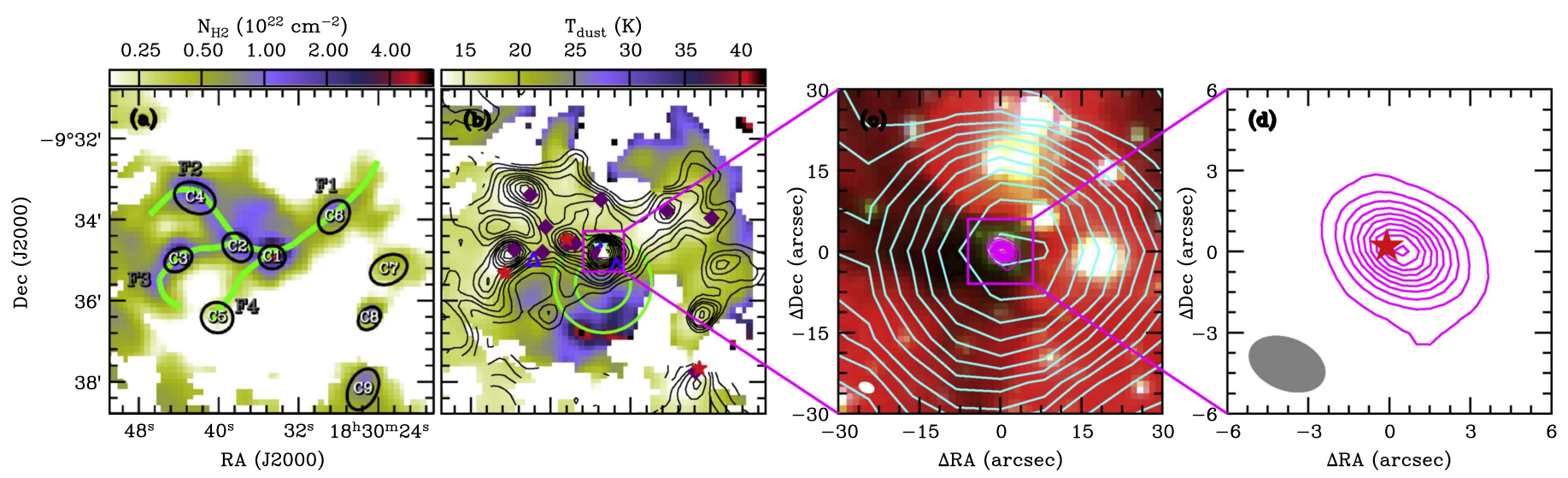

Figure 2. (a) Column density map from SED fitting. The eight clumps, which have been identified based on this map and designated to be C1-C8, are shown as open ellipses. Also labeled are the visually identified filaments F1-F4. (b) Dust temperature map from SED fitting with column density overlaid as contours in levels of $(0.2,0.3,0.4,0.5,0.7,0.9,1.1,1.3,1.6,1.9,2.3,2.8,3.4,4.1,5.0,6.1) \times 10^{22} \mathrm{~cm}^{-1}$. Infrared dark clouds from Peretto \& Fuller (2009) are marked as purple diamonds. The red stars represent young stellar object candidates from Robitaille et al. (2008). EGO G022.04+0.22 is shown as a filled triangle. The three blue open triangles represent molecular hydrogen emission objects from Ioannidis \& Froebrich (2012). The cyan filled inverted triangle labels IRAS 18278-0936. The two green ellipses delineate the inner and outer rings of the infrared bubble MWP1G022027+002159 (Simpson et al. 2012). (c) $\mathrm{H}_{2}$ column density from SED fits (cyan contours) and SMA $1.3 \mathrm{~mm}$ continuum emission (magenta contours) overlaid on an IRAC three-color image with emission at 8.0, 4.5, and 3.6 $\mu \mathrm{m}$ rendered in red, green, and blue, respectively. Cyan contours represent $\mathrm{H}_{2}$ column densities of $(0.7,0.9,1.1,1.3,1.6,1.9,2.3,2.8,3.4,4.1,5.0,6.1) \times 10^{22} \mathrm{~cm}^{-1}$. Magenta contour levels at the center (more visible in (d)) start from $10 \sigma$ and increase with a step of $10 \sigma$, where $1 \sigma=3.2 \mathrm{mJy}^{-1}$ beam ${ }^{-1}$. (d) A close-up view of the SMA $1.3 \mathrm{~mm}$ continuum. The single core is designated as SMA1. A filled star represents the MIR source SSTGLMC G022.0387+00.2222 (MIR1) from the GLIMPSE survey.

Under local thermodynamic equilibrium (LTE) conditions, the column density of a linear molecule can be expressed as

$$
\begin{aligned}
N= & \frac{3 k}{8 \pi^{3} \nu \mu^{2} S} \frac{Q_{\text {rot }}}{g_{J+1}} \exp \left(\frac{E_{\text {up }}}{k T_{\text {ex }}}\right) J\left(T_{\text {ex }}\right) \\
& \times \frac{1}{J\left(T_{\text {ex }}\right)-J\left(T_{\mathrm{bg}}\right)} \frac{\tau}{1-\exp (-\tau)} \int T_{\mathrm{r}} d v .
\end{aligned}
$$

Here, $\mu$ is the permanent dipole moment. For the 1-0 transition, the line strength $S=\frac{J+1}{2 J+3}=\frac{1}{3}$, the degeneracy $g_{\mathrm{J}+1}=$ $2 J+3=3$, where $J$ is the rotational quantum number of the lower state. We have followed McDowell (1988) to write the partition function as $Q_{\text {rot }}=\frac{k T}{h B}+\frac{1}{3}$ where $B$ is the rotational constant.

In the calculation, a single excitation temperature was assumed to be $20 \mathrm{~K}$, which is approximately the averaged dust temperature of the cloud. The $\mathrm{H}_{2}$ column density in each pixel was obtained by assuming a ${ }^{12} \mathrm{C} /{ }^{13} \mathrm{C}=50$ isotope ratio (estimated using Equation (4) of Pineda et al. 2013) and a canonical CO abundance of $X_{\mathrm{CO}}=10^{-4}$. The resultant column densities range from $3.4 \times 10^{21}$ to $2.19 \times 10^{22} \mathrm{~cm}^{-2}$ with a mean of $9.39 \times 10^{21} \mathrm{~cm}^{-2}$. Integrating the whole density map resulted in a total mass of $6117 M_{\odot}$. We note that ${ }^{13} \mathrm{CO}(1-0)$ is assumed to be optically thin and the resultant cloud mass might be the lower limit.

\section{Results of SMA Observations}

\section{1. $1.3 \mathrm{~mm}$ Continuum Emission}

A zoomed-in IRAC three-color image of $\mathrm{C} 1$, the most massive clump in G22, is shown in Figure 2(c) with $N_{\mathrm{H}_{2}}$ and the SMA $1.3 \mathrm{~mm}$ continuum emission overlaid as cyan and magenta contours. With the current SMA angular resolution and sensitivity, only one $1.3 \mathrm{~mm}$ continuum core has been detected at the center and designated as SMA1.
Table 1

Properties of Filaments

\begin{tabular}{lcccc}
\hline \hline Name & $\begin{array}{c}\text { Length } \\
(\mathrm{pc})\end{array}$ & $\begin{array}{c}\text { Mass } \\
\left(M_{\odot}\right)\end{array}$ & $\begin{array}{c}M_{\text {line }} \\
\left(M_{\odot} \mathrm{pc}^{-1}\right)\end{array}$ & $\begin{array}{c}N_{\mathrm{H}_{2}}{ }^{\mathrm{a}} \\
\left(10^{21} \mathrm{~cm}^{-2}\right)\end{array}$ \\
\hline F1 & 2.91 & 357 & 123 & 4.59 \\
F2 & 2.62 & 575 & 220 & 7.28 \\
F3 & 3.02 & 485 & 161 & 7.72 \\
F4 & 1.88 & 102 & 54 & 3.07 \\
\hline
\end{tabular}

Note.

${ }^{\mathrm{a}}$ Averaged column density.

A close-up view of SMA1 is shown in Figure 2(d). 2D Gaussian fitting of the $1.3 \mathrm{~mm}$ continuum emission resulted in an ellipse with a major axis of 3". 68 , a minor axis of 2!" 69 and a position angle of $56^{\circ} .87$. The deconvolved major and minor axes are $2 . " 34$ and 1 ." 70 , smaller than the synthesized beam $(2$ !' $95 \times 1$ !"92). The equivalent angular size of the core is 1 !" 99 , corresponding to a physical diameter of $0.034 \mathrm{pc}(\sim 7000 \mathrm{au})$. The flux density of the core is about $562 \mathrm{mJy}$ with a peak intensity of $350 \mathrm{mJy}^{\text {beam }}{ }^{-1}$.

Assuming the $1.3 \mathrm{~mm}$ continuum emission is optically thin, the core dust mass can be obtained via $M=R_{\mathrm{gd}} S_{\nu} d^{2} /\left[\kappa_{\nu} B_{\nu}(T)\right]$, where $S_{\nu}$ is the observed flux density, $d$ is the distance and $\kappa_{\nu}=1.0 \mathrm{~cm}^{2} \mathrm{~g}^{-1}$ is the dust opacity (Ossenkopf \& Henning 1994). Using the rotational temperature of $\mathrm{CH}_{3} \mathrm{OH}(\sim 227 \mathrm{~K}$, see Section 5.3.1) as the dust temperature, a core mass of $10.4 M_{\odot}$ is reached. The source-averaged $\mathrm{H}_{2}$ number density is about $7.2 \times 10^{6} \mathrm{~cm}^{-3}$. The beam-averaged column density was estimated to be about $1.55 \times 10^{23} \mathrm{~cm}^{-2}$ via $N_{\mathrm{H}_{2}}=$ $I_{\nu} R_{\mathrm{gd}} /\left[\kappa_{\nu} B_{\nu}(T) m_{\mathrm{H}} \mu_{\mathrm{H}_{2}}\right]$.

\subsection{Line Emission}

Figure 4 shows the full band spectra toward the SMA1 peak. Cross-checking the rest frequencies with the Splatalogue molecular database, we have identified 103 emission lines with peaks $>3 \sigma$ where $1 \sigma=80 \mathrm{mJy}^{\text {beam }}{ }^{-1}$. These transitions are 
Table 2

Properties of the Identified Dense Clumps

\begin{tabular}{|c|c|c|c|c|c|c|c|c|c|c|c|c|}
\hline Clulmp & R.A. & Decl. & $\begin{array}{c}\Theta_{\text {maj }} \\
\left({ }^{\prime \prime}\right)\end{array}$ & $\begin{array}{c}\Theta_{\min } \\
\left({ }^{\prime \prime}\right)\end{array}$ & $\begin{array}{l}\text { PA } \\
\left({ }^{\circ}\right)\end{array}$ & $\begin{array}{c}\text { FWHM }^{\mathrm{a}} \\
\left({ }^{\prime \prime}\right)\end{array}$ & $\begin{array}{l}r_{\mathrm{eq}}^{\mathrm{b}} \\
(\mathrm{pc})\end{array}$ & $\begin{array}{l}T_{\text {dust }} \\
(\mathrm{K})\end{array}$ & $\begin{array}{c}N_{\mathrm{H}_{2}} \\
\left(10^{22} \mathrm{~cm}^{-2}\right)\end{array}$ & $\begin{array}{c}M_{\mathrm{cl}} \\
\left(M_{\odot}\right)\end{array}$ & $\begin{array}{c}\Sigma_{\text {mass }} \\
\left(\mathrm{g} \mathrm{cm}^{-2}\right)\end{array}$ & $\left(10^{4} \mathrm{~cm}^{n_{\mathrm{H}_{2}}}\right)$ \\
\hline $\mathrm{C} 1$ & $18^{\mathrm{h}} 30^{\mathrm{m}} 34^{\mathrm{s}} \cdot 64$ & $-9^{\mathrm{d}} 34^{\mathrm{m}} 55^{\mathrm{s}} .9$ & 38.8 & 34.6 & 90.3 & 36.5 & 0.39 & 20.9 & 6.55 & 590 & 0.26 & 3.52 \\
\hline $\mathrm{C} 2$ & $18^{\mathrm{h}} 30^{\mathrm{m}} 38^{\mathrm{s}} \cdot 12$ & $-9^{\mathrm{d}} 34^{\mathrm{m}} 41^{\mathrm{s}} \cdot 1$ & 48.1 & 37.8 & 49.2 & 42.3 & 0.49 & 18.4 & 2.39 & 319 & 0.09 & 0.91 \\
\hline $\mathrm{C} 3$ & $18^{\mathrm{h}} 30^{\mathrm{m}} 44^{\mathrm{s}} \cdot 04$ & $-9^{\mathrm{d}} 34^{\mathrm{m}} 59^{\mathrm{s}} .7$ & 43.9 & 32.1 & 123.4 & 36.8 & 0.39 & 13.8 & 2.68 & 270 & 0.12 & 1.56 \\
\hline $\mathrm{C} 4$ & $18^{\mathrm{h}} 30^{\mathrm{m}} 42^{\mathrm{s}} \cdot 38$ & $-9^{\mathrm{d}} 33^{\mathrm{m}} 28^{\mathrm{s}} \cdot 0$ & 62.9 & 44.3 & 67.2 & 52.4 & 0.67 & 14.8 & 2.17 & 409 & 0.06 & 0.48 \\
\hline $\mathrm{C} 5$ & $18^{\mathrm{h}} 30^{\mathrm{m}} 40^{\mathrm{s}} \cdot 15$ & $-9^{\mathrm{d}} 36^{\mathrm{m}} 25^{\mathrm{s}} .2$ & 47.4 & 45.0 & 66.3 & 46.2 & 0.56 & 23.5 & 0.41 & 62 & 0.01 & 0.12 \\
\hline C6 & $18^{\mathrm{h}} 30^{\mathrm{m}} 28^{\mathrm{s}} \cdot 44$ & $-9^{\mathrm{d}} 33^{\mathrm{m}} 56^{\mathrm{s}} .0$ & 53.9 & 40.2 & 139.6 & 46.1 & 0.56 & 14.0 & 1.11 & 174 & 0.04 & 0.34 \\
\hline $\mathrm{C} 7$ & $18^{\mathrm{h}} 30^{\mathrm{m}} 22^{\mathrm{s}} .97$ & $-9^{\mathrm{d}} 35^{\mathrm{m}} 14^{\mathrm{s}} .0$ & 57.6 & 41.7 & 116.0 & 48.5 & 0.60 & 19.3 & 0.54 & 96 & 0.02 & 0.15 \\
\hline $\mathrm{C} 8$ & $18^{\mathrm{h}} 30^{\mathrm{m}} 24^{\mathrm{s}} .90$ & $-9^{\mathrm{d}} 36^{\mathrm{m}} 26^{\mathrm{s}} \cdot 3$ & 38.0 & 29.0 & 137.0 & 32.3 & 0.30 & 15.9 & 0.98 & 79 & 0.06 & 1.03 \\
\hline C9 & $18^{\mathrm{h}} 30^{\mathrm{m}} 25^{\mathrm{s}} \cdot 54$ & $-9^{\mathrm{d}} 38^{\mathrm{m}} 11^{\mathrm{s}} .2$ & 63.9 & 42.5 & 154.6 & 51.5 & 0.65 & 15.0 & 1.00 & 190 & 0.03 & 0.24 \\
\hline
\end{tabular}

Notes.

${ }^{\mathrm{a}}$ Full width at half maximum: FWHM $=\sqrt{\Theta_{\text {maj }} \Theta_{\text {min }}}$.

b Equivalent radius: $r_{\text {eq }}=d \sqrt{\Theta_{\text {maj }} \Theta_{\text {min }}-\theta_{\text {beam }}^{2}} / \sqrt{2 \ln 2}$, where $\theta_{\text {beam }}=25^{\prime \prime}$.

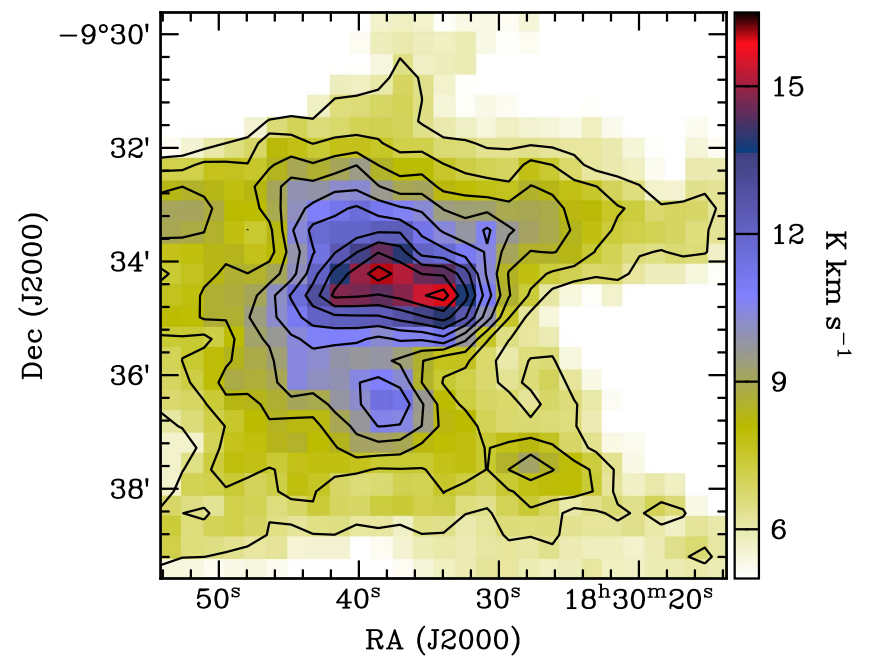

Figure 3. Velocity-integrated intensity map of ${ }^{13} \mathrm{CO}(1-0)$. The velocity interval for integration is $[46,59] \mathrm{km} \mathrm{s}^{-1}$. The contours start from $6.5 \mathrm{~K} \mathrm{~km} \mathrm{~s}^{-1}$ and increase with a step of $1.0 \mathrm{~K} \mathrm{~km} \mathrm{~s}^{-1}$.

from 19 species (26 isotopologues) including nitrogen-bearing species (e.g., $\mathrm{HC}_{3} \mathrm{~N}, \mathrm{CH}_{3} \mathrm{CN}$ ), complex organic molecules (e.g., $\mathrm{CH}_{3} \mathrm{CHO}, \mathrm{CH}_{3} \mathrm{OCH}_{3}$ ), and classical tracers of bulk motions (e.g., $\mathrm{CO}, \mathrm{SiO})$.

We extracted spectra of all detected transitions toward the SMA1 peak and used the GILDAS/CLASS software to perform Gaussian profile fitting to get the line peak intensities, velocity centroids, line widths, and velocity-integrated intensities. The systemic velocity of SMA1 was estimated to be $50.3 \pm$ $0.1 \mathrm{~km} \mathrm{~s}^{-1}$ via averaging line centers of transitions with FWHM $\leqslant 9 \mathrm{~km} \mathrm{~s}^{-1}$ and $I_{\text {peak }} \geqslant 0.4 \mathrm{Jy}_{\text {beam }}{ }^{-1}$.

Inspection of velocity-integrated intensity maps shows that lines from 19 molecules trace a single-core morphology in accord with the $1.3 \mathrm{~mm}$ continuum emission. On the other hand, lines of $\mathrm{CO},{ }^{13} \mathrm{CO}, \mathrm{C}^{18} \mathrm{O}, \mathrm{H}_{2} \mathrm{CO}$, SO, and $\mathrm{SiO}$ reveal extended structures either from the surrounding envelope or entrained outflows (see Section 4.3). Among the 15 detected $\mathrm{CH}_{3} \mathrm{OH}$ lines, 13 trace a single-core spatially coincident with the $1.3 \mathrm{~mm}$ core. The other two methanol transitions show anomalous emission in the vicinity (see Section 4.4).

\subsection{Molecular Outflows}

In multi-object surveys, previous works have detected outflow indicators toward EGO G022.04+0.22 (C1 in our work), including Class I methanol masers (Cyganowski et al. 2009; Chen et al. 2011), "red-skewed" asymmetric line profiles of $\mathrm{CO}$ and $\mathrm{HCO}^{+}$(Rygl et al. 2013), and broad SiO (5-4) emission (Cyganowski et al. 2009). The existence of outflows in $\mathrm{C} 1$ is confirmed by the SMA observations presented in this work.

SMA CO (2-1) shows very broad $\left(>70 \mathrm{~km} \mathrm{~s}^{-1}\right.$ with respect to the systemic velocity) wing emission. Velocity-integrated intensities of the blue and red wings (see Figure 5(a)) reveal two blueshifted and two redshifted lobes (B1, B2, R1 and B2). These outflow lobes can be well traced back to the SMA1 peak. The multiple lobes of the outflow also could be due to the presence of two, unresolved protostellar objects. The outflows are also seen in emission from $\mathrm{SiO}$ and $\mathrm{H}_{2} \mathrm{CO}$ (see Figures 5(b) and (c)). The GLIMPSE point source SSTGLMC G022.0387 +0.2222 (MIR1, hereafter), which is an early-stage high-mass protostar (see Section 5.3) associated with SMA1, could be the driving source.

Copious $44 \mathrm{GHz}$ Class I $\mathrm{CH}_{3} \mathrm{OH}$ masers detected by Cyganowski et al. (2009) are shown in Figure 5 with crosses (" $\times$ "). The spatial distribution of the $44 \mathrm{GHz}$ masers shows four distinct groups. The southern group of masers are distributed mainly along a N-S line closely associated with the R1 lobe. The northeast group of masers is spatially coincident with the B2 lobe and the extended $4.5 \mu \mathrm{m}$ emission. In the north, a crowd of masers are located in between the B1 and B2 lobes. There are also several maser spots on the edge of the B1 lobe. A small group of masers detected in the southwest are not consistent with any high-velocity $\mathrm{CO}$ emission. Noticeably, the velocities of maser spots generally agree with those of the associated outflow lobes, supporting their shockdriven origin. There is a reversal of maser velocities at the southern tip of the R1 lobe coincident with a change of outflow direction from south to the southeast. This intriguing feature could be due to the influence of the adjacent infrared bubble (see Section 5.4).

Physical parameters of the outflows have been calculated from the $\mathrm{CO}$ line wings under LTE conditions and assuming optically thin emission. The CO column density of each pixel 

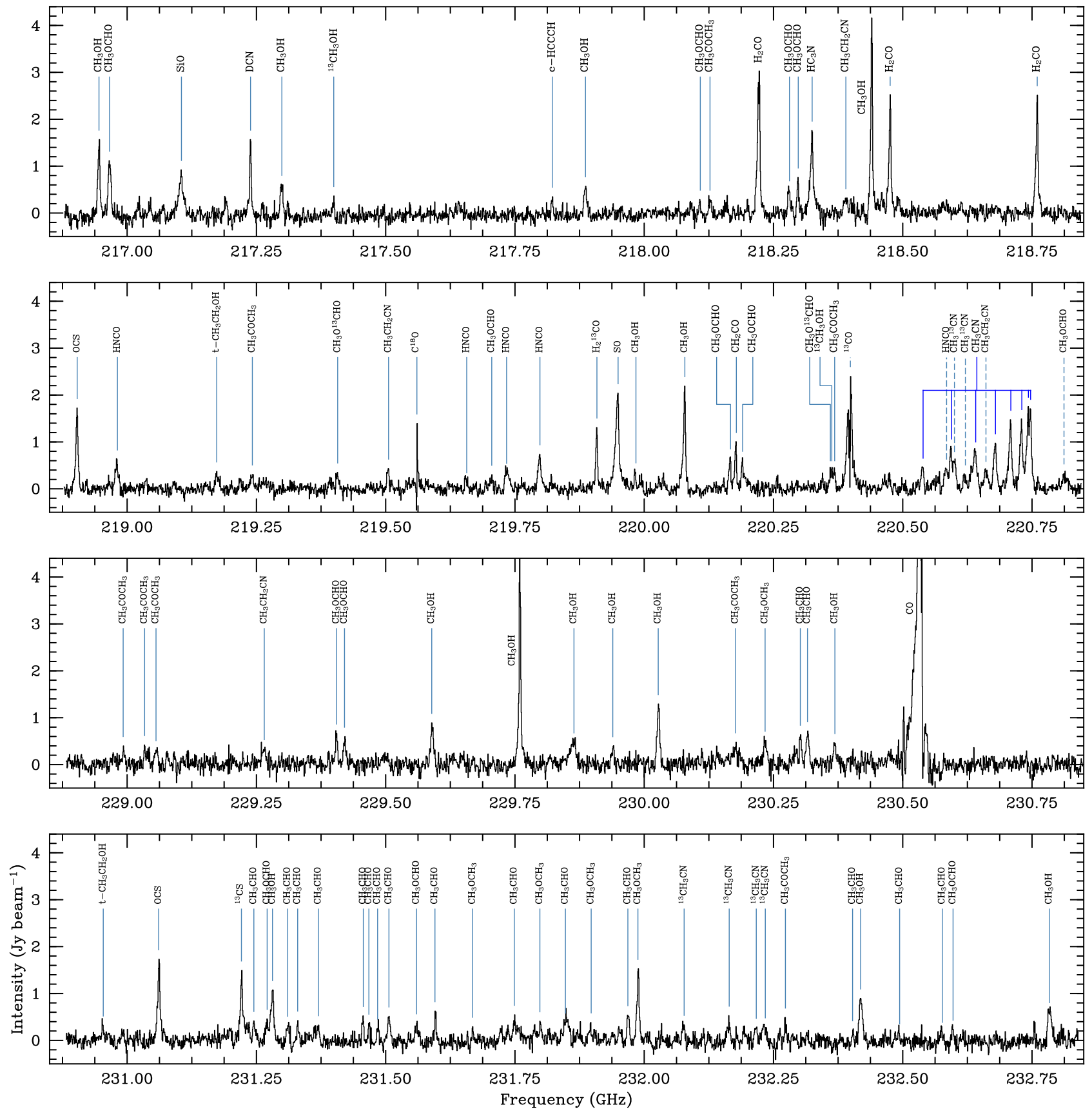

Figure 4. SMA LSB and USB spectra toward the peak of SMA1. Lines with peaks no smaller than $3 \sigma\left(240 \mathrm{mJy}^{\text {beam }}{ }^{-1}\right)$ have been labeled.

in each velocity channel can be straightforwardly derived from Equation (3) as

$$
\begin{aligned}
d N_{\mathrm{CO}}\left(\mathrm{cm}^{-2}\right)= & 2.49 \times 10^{14}\left(T_{\mathrm{ex}}+0.92\right) \exp \left(\frac{17}{T_{\mathrm{ex}}}\right) \\
& \times \frac{J\left(T_{\mathrm{ex}}\right)}{J\left(T_{\mathrm{ex}}\right)-0.018} T_{\mathrm{r}} d v \\
= & F\left(T_{\mathrm{ex}}\right) T_{\mathrm{r}} d v,
\end{aligned}
$$

where $d v$ is the velocity interval in $\mathrm{km} \mathrm{s}^{-1}$ and $T_{\mathrm{r}}$ is the measured brightness temperature in $\mathrm{K}$. In the calculation, the excitation temperature has been assumed to be approximately the source-averaged dust temperature of clump $\mathrm{C} 1$ which is $21 \mathrm{~K}$. The outflow mass, momentum, energy, dynamical age, mass loss rate, and mechanical force have been obtained from

$$
\begin{gathered}
M=\mu_{\mathrm{H}_{2}} m_{\mathrm{H}} F\left(T_{\mathrm{ex}}\right) \frac{d^{2}}{X_{\mathrm{CO}}} \int_{\Omega} \int_{v} T_{\mathrm{r}} d \Omega d v, \\
P=\mu_{\mathrm{H}_{2}} m_{\mathrm{H}} F\left(T_{\mathrm{ex}}\right) \frac{d^{2}}{X_{\mathrm{CO}}} \int_{\Omega} \int_{v} T_{\mathrm{r}} v d \Omega d v, \\
E=\frac{1}{2} \mu_{\mathrm{H}_{2}} m_{\mathrm{H}} F\left(T_{\mathrm{ex}}\right) \frac{d^{2}}{X_{\mathrm{CO}}} \int_{\Omega} \int_{v} T_{\mathrm{r}} v^{2} d \Omega d v, \\
t_{\mathrm{dyn}}=\frac{L_{\mathrm{flow}}}{v_{\text {char }}}, \\
\dot{M}_{\mathrm{out}}=\frac{M}{t_{\mathrm{dyn}}},
\end{gathered}
$$




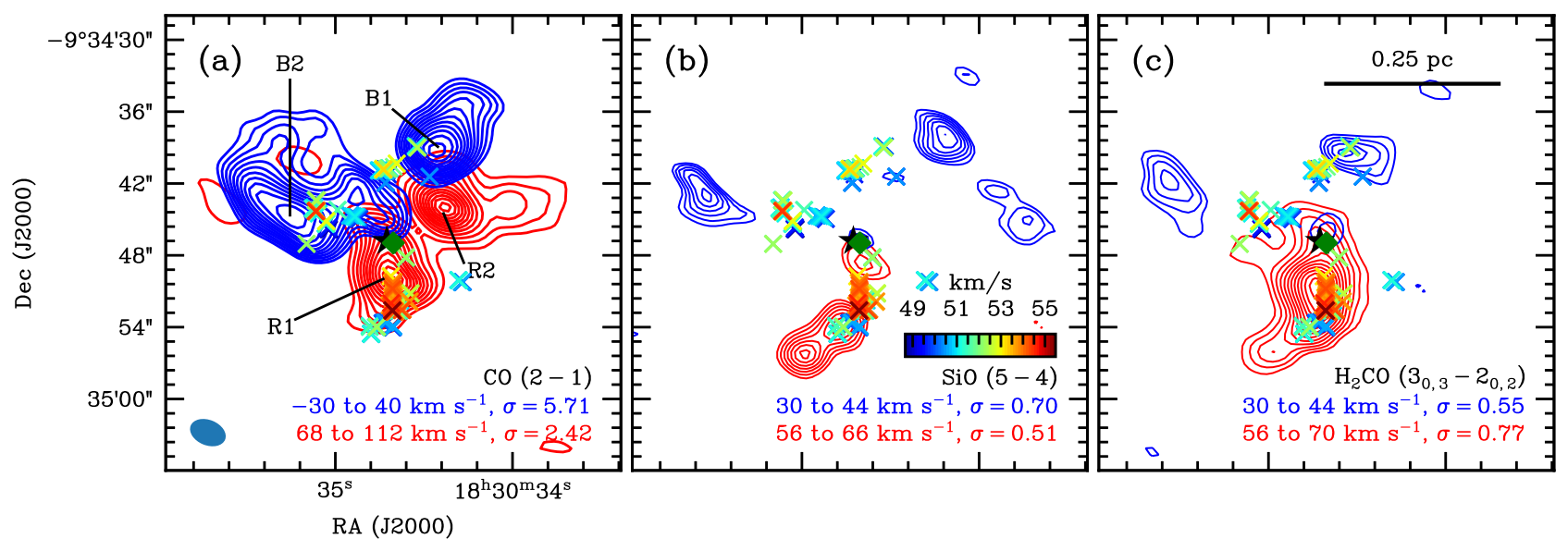

Figure 5. Outflows revealed by $\mathrm{CO}(2-1)(\mathrm{a}), \mathrm{SiO}(5-4)(\mathrm{b})$, and $\mathrm{H}_{2} \mathrm{CO}\left(3_{0,3}-2_{0,2}\right)(\mathrm{c})$. Velocity intervals and $1 \sigma$ noise levels of the red and blue lobes for each line are labeled in the bottom-right corner of each panel. For $\mathrm{CO}(2-1)$, the blue contours start from $3 \sigma$ and increase by $2 \sigma$, the blue contours start from $3 \sigma$ and increase by $1 \sigma$. For $\mathrm{SiO}(5-4)$, the blue contours start from $3 \sigma$ and increase by $1 \sigma$, the blue contours start from $3 \sigma$ and increase by $0.5 \sigma$. For $\mathrm{H}_{2} \mathrm{CO}\left(3_{0,3}-2_{0,2}\right)$, the blue contours start from $3 \sigma$ and increase by $1 \sigma$, the blue contours start from $3 \sigma$ and increase $1 \sigma$. The $44 \mathrm{GHz}$ Class I methanol masers from Cyganowski et al. (2009) are marked with crosses and color-coded according to their velocities. The green diamond and the black star show the locations of the 1.3 mm core SMA1 and embedded protostar SSTGLMC G022.0387+00.2222 (MIR1).

$$
F_{\text {mech }}=\frac{P}{t_{\text {dyn }}}
$$

Here, $\Omega$ is the total solid angle that the flow subtends, $v$ is the flow velocity with respect to the systemic velocity, $v_{\text {char }}=$ $P / M$ is the characteristic outflow velocity, and $L_{\text {flow }}$ is the flow length.

The resultant outflow parameters are given in Table 3 . The four lobes have dynamical ages ranging from $0.47 \times 10^{4}$ to $1.33 \times 10^{4}$ years, comparable to those of other outflows in early high-mass star-forming regions, e.g., G240.31+0.07 $\left(2.4 \times 10^{4}\right.$ years, Qiu et al. 2009), G24.78+0.08 $(\sim 2 \times$ $10^{4}$ years, Beltrán et al. 2011), G28.34+0.06 ((1.3-3.4) $\times$ $10^{4}$ years, Wang et al. 2011), G11.11-0.12 $\left(\sim 2.5 \times 10^{4}\right.$ years, Wang et al. 2014), and G9.62+0.19 MM6 $\left(8.5 \times 10^{3}\right.$ years, Liu et al. 2017). The relatively small dynamical ages are also consistent with the stellar age estimate from SED fitting $\left([0.6-3.8] \times 10^{4}\right.$ years, see Section 5.3.2).

\subsection{Millimeter Methanol Maser Emission}

Figures 6(a)-(o) show the velocity-integrated intensity maps of all 15 detected transitions of $\mathrm{CH}_{3} \mathrm{OH}$ with upper energies ranging from 40 to $802 \mathrm{~K}$. All $\mathrm{CH}_{3} \mathrm{OH}$ transitions, with the exception of the $4_{2,2}-3_{1,2} \quad E$ (at $218.440 \mathrm{GHz}$ with $E_{\text {up }} / k=46 \mathrm{~K}$ ) and $8_{-1,8}-7_{0,7} \quad E$ (at $229.759 \mathrm{GHz}$ with $E_{\text {up }} / k=89 \mathrm{~K}$ ), show single-core morphologies. Emission from the $4_{2,2}-3_{1,2} E$ and $8_{-1,8}-7_{0,7} E$ transitions reveals more complex structures. Figure 6 shows maps of the four $\mathrm{CH}_{3} \mathrm{OH}$ transitions with the lowest upper energies. Compared to the other methanol transitions, strong $\mathrm{CH}_{3} \mathrm{OH}\left(8_{-1,8}-7_{0,7}\right)$ and $\mathrm{CH}_{3} \mathrm{OH}\left(4_{2,2}-3_{1,2}\right)$ emission can be detected to the north, south, and northeast of the $1.3 \mathrm{~mm}$ core SMA1, spatially coincident with Class I $44 \mathrm{GHz}$ methanol masers, suggestive of maser emission in the $\mathrm{CH}_{3} \mathrm{OH}\left(8_{-1,8}-7_{0,7}\right)$ and $\mathrm{CH}_{3} \mathrm{OH}\left(4_{2,2}-3_{1,2}\right)$ transitions.

$\mathrm{CH}_{3} \mathrm{OH}\left(8_{-1,8}-7_{0,7}\right)$ and $\mathrm{CH}_{3} \mathrm{OH}\left(4_{2,2}-3_{1,2}\right)$ spectra toward core SMA1, and positions A, B, and C marked in Figures 6(b) and (d) are presented in Figures 6(p)-(s). For comparison, spectra of $\mathrm{CH}_{3} \mathrm{OH}\left(3_{-2,2}-4_{-1,4}\right)$ at $230.027 \mathrm{GHz}$ with
Table 3

Outflow Parameters

\begin{tabular}{|c|c|c|c|c|c|c|c|}
\hline Lobe & $L_{\text {flow }}$ & $\left(\mathrm{km} \mathrm{s}^{-1}\right)$ & $M_{\text {out }}$ & $\begin{array}{c}P_{\text {out }} \\
\left(M_{\odot} \mathrm{km}\right. \\
\left.\mathrm{s}^{-1}\right)\end{array}$ & $\begin{array}{l}E_{\text {out }} \\
\left(M_{\odot}\right. \\
\mathrm{km}^{2} \\
\left.\mathrm{~s}^{-2}\right)\end{array}$ & $\begin{array}{c}t_{\mathrm{dyn}} \\
\left(10^{4}\right. \\
\text { years })\end{array}$ & $\begin{array}{c}\dot{M}_{\text {out }_{5}} \\
\left(10^{-5}\right. \\
M_{\odot} \\
\left.\mathrm{yr}^{-1}\right)\end{array}$ \\
\hline B1 & 0.26 & 22.47 & 0.57 & 12.96 & 182.94 & 1.12 & 5.16 \\
\hline B2 & 0.27 & 19.63 & 1.23 & 24.22 & 300.82 & 1.33 & 9.25 \\
\hline $\mathrm{R} 1$ & 0.13 & 27.43 & 0.37 & 10.21 & 158.28 & 0.47 & 7.95 \\
\hline $\mathrm{R} 2$ & 0.26 & 28.18 & 0.38 & 10.71 & 170.64 & 0.89 & 4.29 \\
\hline tal & $\ldots$ & $\cdots$ & 2.56 & 58.10 & 812.68 & $\cdots$ & 26.65 \\
\hline
\end{tabular}

$E_{\text {up }} / k=40 \mathrm{~K}$ and $\mathrm{CH}_{3} \mathrm{OH}\left(5_{1,4}-4_{2,2}\right)$ at $216.946 \mathrm{GHz}$ with $E_{\text {up }} / k=56 \mathrm{~K}$ are also shown. $\mathrm{CH}_{3} \mathrm{OH}\left(8_{-1,8}-7_{0,7}\right)$ and $\mathrm{CH}_{3} \mathrm{OH}\left(4_{2,2}-3_{1,2}\right)$ spectra at position $\mathrm{A}$ are narrow, resembling features of maser emission. Relatively broader spectra at positions $\mathrm{B}$ and $\mathrm{C}$ may be due to the blending of unresolved maser spots.

The peak intensities of $\mathrm{CH}_{3} \mathrm{OH}\left(8_{-1,8}-7_{0,7}\right)$ at positions SMA1, A, B, and C are 4.9, 3.5, 3.5, and $1.1 \mathrm{Jy}^{-1}$ beam $^{-1}$. For $\mathrm{CH}_{3} \mathrm{OH}\left(4_{2,2}-3_{1,2}\right)$, the peak intensities at SMA1, A, B, and $\mathrm{C}$ are $4.1,2.9,2.5$, and $1.4 \mathrm{Jy}_{\text {beam }}{ }^{-1}$. The peak intensities of $\mathrm{CH}_{3} \mathrm{OH}\left(3_{-2,2}-4_{-1,4}\right)$ at SMA1, A, B, and $\mathrm{C}$ are 1.4, 0.4, 0.2 , and $0.1 \mathrm{Jy}$ beam $^{-1}$. The $8_{-1,8}-7_{0,7} / 3_{-2,2}-4_{-1,4}$ line ratio has previously been used as a discriminant between thermal and non-thermal $8_{-1,8}-7_{0,7}$ emission, with ratios $>3$ indicative of non-thermal (Slysh et al. 2002; Cyganowski et al. 2011). The $8_{-1,8}-7_{0,7} / 3_{-2,2}-4_{-1,4}$ ratios for SMA1, A, $\mathrm{B}$, and $\mathrm{C}$ are $3.5,8.8,17.5$, and 11.0 , suggestive of nonthermal $8_{-1,8}-7_{0,7}$ emission at positions $\mathrm{A}, \mathrm{B}$, and $\mathrm{C}$. We can similarly use the $4_{2,2}-3_{1,2} / 3_{-2,2}-4_{-1,4}$ line ratio to distinguish thermal from non-thermal $4_{2,2}-3_{1,2}$ emission. Under LTE and optically thin conditions, the thermal emission ratio of two $\mathrm{CH}_{3} \mathrm{OH}$ transitions can be obtained from

$$
R\left(\frac{\nu_{1}}{\nu_{2}}\right)=\left(\frac{A_{\mathrm{ul1}}}{A_{\mathrm{ul} 2}}\right)\left(\frac{\nu_{2}}{\nu_{1}}\right)^{2} \exp \left(\frac{E_{\mathrm{up} 2}-E_{\mathrm{up} 1}}{k T}\right)
$$



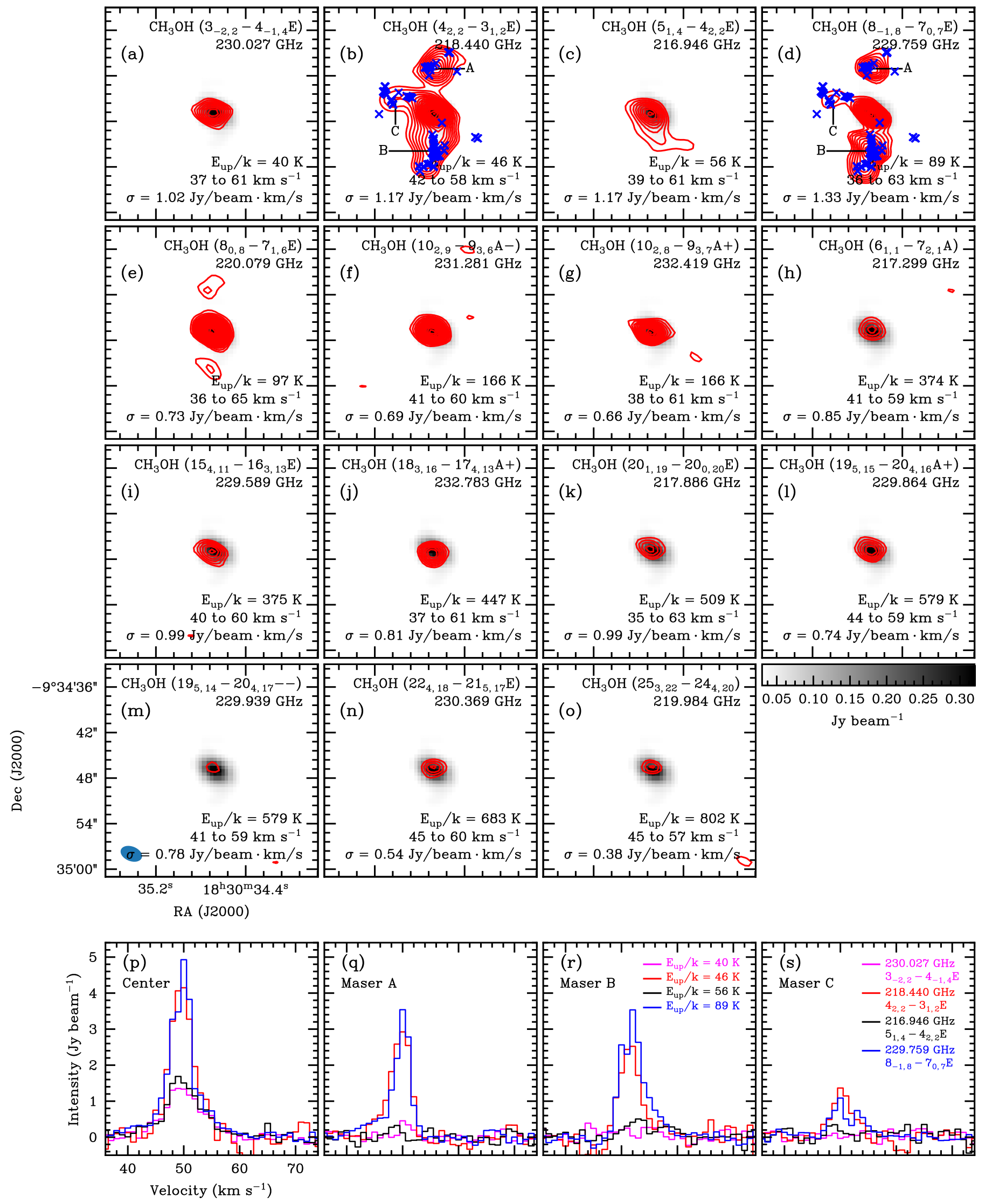

Figure 6. (a)-(o): Velocity integrated intensities (contours) of all $15 \mathrm{CH}_{3} \mathrm{OH}$ transitions with the lowest upper energies overlaid on the $1.3 \mathrm{~mm}$ continuum image. Contours for each line start from $3 \sigma$ with an increment of $1 \sigma$. The relevant transitions, velocity intervals for integration, and $1 \sigma$ noise levels are labeled at the bottom individual panels. Panels (p)-(s) presents spectra of $\mathrm{CH}_{3} \mathrm{OH}\left(3_{-2,2}-4_{-1,4}\right), \mathrm{CH}_{3} \mathrm{OH}\left(4_{2,2}-3_{1,2}\right), \mathrm{CH}_{3} \mathrm{OH}\left(5_{1,4}-4_{2,2}\right)$, and $\mathrm{CH}_{3} \mathrm{OH}\left(8_{-1,8}-7_{0,7}\right)$ at core $\mathrm{SMA} 1$, and positions $\mathrm{A}, \mathrm{B}$, and $\mathrm{C}$ labeled in (b) and (d). 
With an assumed temperature of $21 \mathrm{~K}$ (similar to the dust temperature of clump $\mathrm{C} 1$ ) deviating from the $1.3 \mathrm{~mm}$ core, the thermal emission $4_{2,2}-3_{1,2} / 3_{-2,2}-4_{-1,4}$ ratio will be approximately 3 . Thus, we suggest a ratio threshold of 3 as a demarcation of thermal and non-thermal $218.440 \mathrm{GHz}$ emission. The $4_{2,2}-3_{1,2} / 3_{-2,2}-4_{-1,4}$ ratios for SMA1, A, B, and $\mathrm{C}$ are $2.9,7.3,12.5$, and 14.0, supportive of non-thermal $218.440 \mathrm{GHz}$ emission at positions $\mathrm{A}, \mathrm{B}$, and $\mathrm{C}$. The $\mathrm{CH}_{3} \mathrm{OH}\left(8_{-1,8}-7_{0,7}\right)$ and $\mathrm{CH}_{3} \mathrm{OH}\left(4_{2,2}-3_{1,2}\right)$ emission features at positions $\mathrm{A}, \mathrm{B}$, and $\mathrm{C}$ spatially coincide well with $44 \mathrm{GHz}$ Class $\mathrm{I} \mathrm{CH}_{3} \mathrm{OH}$ masers. This agreement, along with large $8_{-1,8}-7_{0,7} / 3_{-2,2}-4_{-1,4}$ and $4_{2,2}-3_{1,2} / 3_{-2,2}-4_{-1,4}$ ratios and narrow line widths (especially for position $\mathrm{A}$ ), gives strong support to the interpretation of millimeter $\mathrm{CH}_{3} \mathrm{OH}$ maser emission.

Maser emission of $\mathrm{CH}_{3} \mathrm{OH}\left(8_{-1,8}-7_{0,7}\right)$ at $229.759 \mathrm{GHz}$ has been discovered by Slysh et al. (2002) in DR21(OH) and DR21 West, and reported in HH 80-81 (Qiu \& Zhang 2009), IRAS 05345+3157 (Fontani et al. 2009), NGC 7538 (Qiu et al. 2011), and EGOs G11.92-0.61, G18.67+0.03 and G19.01-0.03 (Cyganowski et al. 2011, 2012). However, maser emission of the $\mathrm{CH}_{3} \mathrm{OH}\left(4_{2,2}-3_{1,2}\right)$ transition at $218.440 \mathrm{GHz}$ has, to our knowledge, only been tentatively detected in DR21 $(\mathrm{OH})$ by Zapata et al. (2012) and in NGC $6334 \mathrm{I}(\mathrm{N})$ by Hunter et al. (2014) prior to this work. Population inversion for the $218.440 \mathrm{GHz}$ transition has been predicted in maser models (Voronkov et al. 2012). The $J_{2}-(J-1)_{1}$ transition and association with $44 \mathrm{GHz}$ Class I masers suggest that the $218.440 \mathrm{GHz}$ $\mathrm{CH}_{3} \mathrm{OH}$ masers detected in $\mathrm{G} 22$ are Class I type. The small number of known sources exhibiting maser emission in this transition is likely to be because few previous observations of high-mass star formation regions have covered this line.

\section{Discussion}

\subsection{Global Collapse of the Cloud}

\subsubsection{Density Structure}

The probability distribution function (PDF) of $N_{\mathrm{H}_{2}}$ is shown in Figure 7(a). The high-density end of the $N_{\mathrm{H}_{2}}-\mathrm{PDF}$ of G22 can be fitted to a power-law. Such $\mathrm{N}_{\mathrm{H}_{2}}-\mathrm{PDF}$ profiles have been reported in many IRDCs and IR-bright clouds (e.g., Schneider et al. 2015a, and references therein). Power-law tails in $\mathrm{N}_{\mathrm{H}_{2}}-$ PDFs also have been observed in numerical works (e.g., Federrath et al. 2008; Klessen \& Hennebelle 2010; Kritsuk et al. 2011). In both theoretical and observational studies, selfgravity has been suggested to be the dominant process in the formation of the power-law tail. Assuming a spherical symmetry, the power-law slope $m$ of the $N_{\mathrm{H}_{2}}-\mathrm{PDF}$ is related to the exponent $\alpha$ of a radial density profile of $\rho(r) \propto r^{-\alpha}$ and $\alpha=-2 / m+1$ (Federrath \& Klessen 2013). An $\alpha$ of about $1.81 \pm 0.23$ is consistent with self-gravity (Girichidis et al. 2014; Schneider et al. 2015b). Such self-gravity can be attributed to local free-fall of individual clumps/cores or global collapse.

Figure 7(b) shows the radial column density profile, which can be well fitted with a power-law $N \propto \rho(r) \times$ $r \propto r^{1-\alpha} \propto r^{\gamma}$. Here, $\alpha$ is the exponent of the underlying radial number density profile. The resultant $\alpha=1.80 \pm 0.10$ is comparable to the $\alpha$ from the power-law tail of the $N_{\mathrm{H}_{2}}-\mathrm{PDF}$, consistent with gravitational collapse on large scales. This
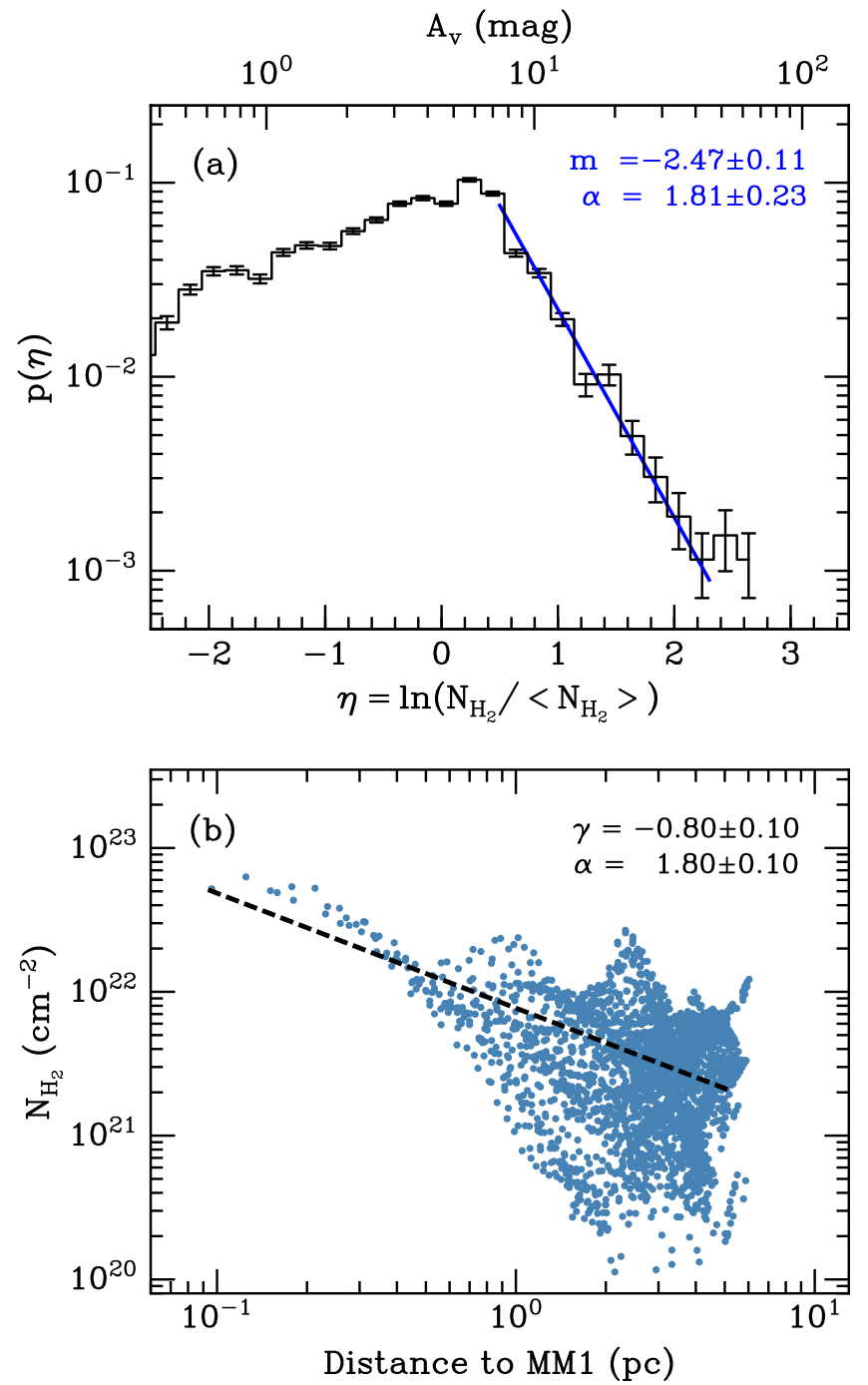

Figure 7. (a) Probability distribution function (PDF) of $N_{\mathrm{H}_{2}}$. The blue solid line shows a power-law fit to the high-column density part of the PDF. Here, $\left\langle N_{\mathrm{H}_{2}}\right\rangle=4.2 \times 10^{21} \mathrm{~cm}^{-2}$. A conversion factor of $N_{\mathrm{H}_{2}} / A_{\mathrm{v}}=9.4 \times$ $10^{20} \mathrm{~cm}^{-2} \mathrm{mag}^{-1}$ is used to convert column density to visual extinction. (b) Radial column density profile. Each point represents one pixel value in the map. The $x$-axis gives the distance to clump $\mathrm{C} 1$. The dashed line shows a power-law fit. The exponent $\gamma$ and the exponent $(\alpha)$ of the underlying radial number density profile $\rho \propto r^{-\alpha} \propto r^{\gamma-1}$ are also labeled.

scenario is further supported by large-scale velocity gradients discussed in the following section.

\subsubsection{Filamentary Collapse}

Assuming cylindrical hydrostatic equilibrium, a critical mass per unit length $M_{\text {line }}$ has been proposed to be $M_{\text {line,crit }}=$ $2 c_{\mathrm{s}}^{2} / G=16.7\left(\frac{T}{10 \mathrm{~K}}\right) M_{\odot} \mathrm{pc}^{-2}$ (Ostriker 1964; Inutsuka \& Miyama 1997; Kirk et al. 2013; André et al. 2014). With a typical temperature of about $20 \mathrm{~K}$ (see Section 3.1 and Figure 2), the critical $M_{\text {line,crit }}$ value is about $33 M_{\odot} \mathrm{pc}^{-1}$ for the filaments in G22. All four filaments are supercritical with $M_{\text {line }}$ significantly larger than the critical value, consistent with the velocity measurements showing that gas is globally infalling in these filaments (see bellow). The mass per unit length for the four filaments ranges from 54 to $220 M_{\odot} \mathrm{pc}^{-1}$ (see Table 1 ). The $M_{\text {line }}$ of filaments in G22 are comparable to those in DR21 where gas is 


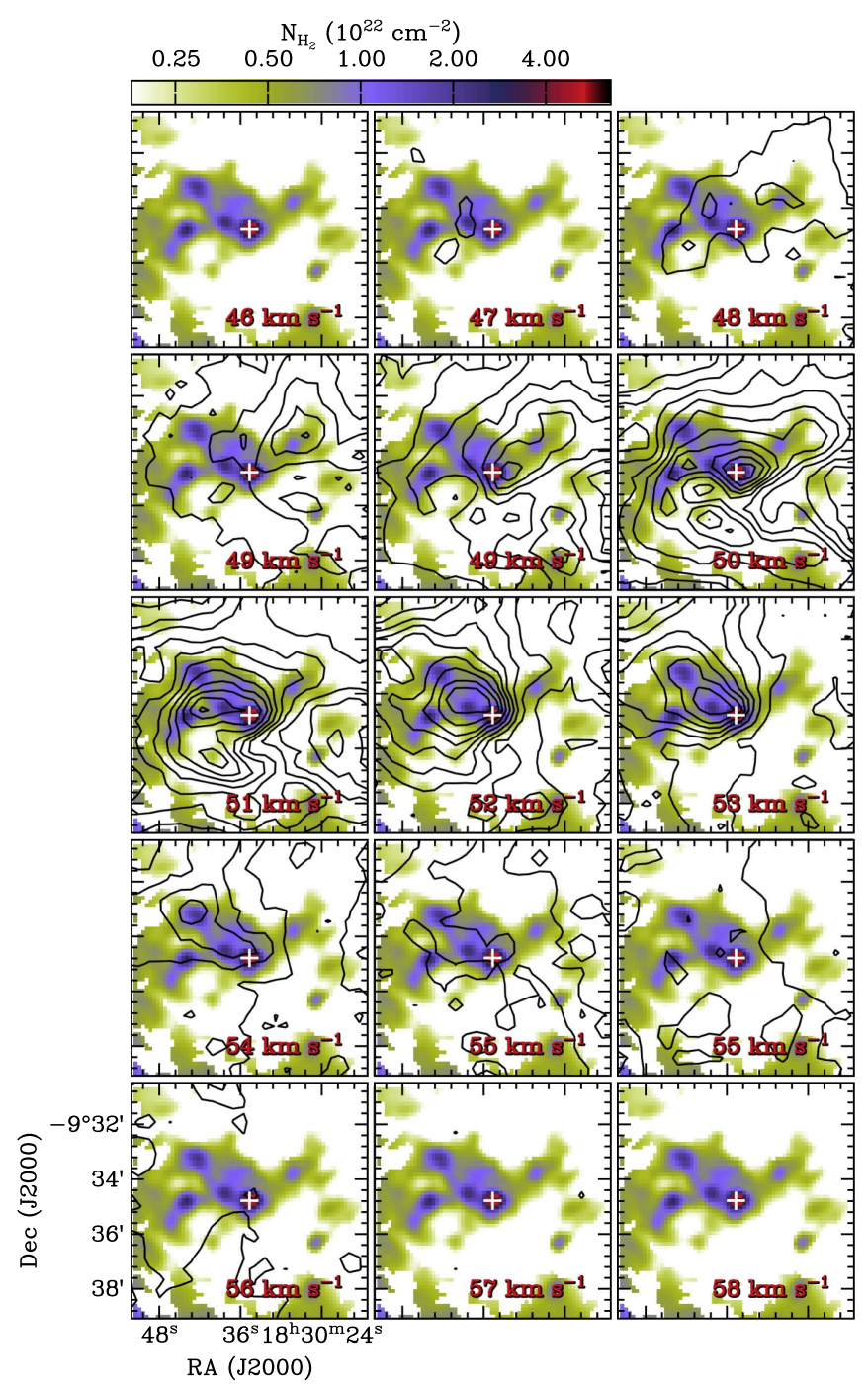

Figure 8. GRS ${ }^{13} \mathrm{CO}(1-0)$ channel maps overlaid on the column density map from SED fits. The velocity interval of integration for each subplot covers $1 \mathrm{~km} \mathrm{~s}^{-1}$, with the center value marked in the lower-right corner. ${ }^{13} \mathrm{CO}$ contours start from $4 \sigma$ and increase with a step of $7 \sigma$. Here, $1 \sigma=0.05 \mathrm{~K} \mathrm{~km} \mathrm{~s}^{-1}$.

channeled along the filaments onto the central ridge region (Hennemann et al. 2012).

GRS ${ }^{13} \mathrm{CO}(1-0)$ channel maps are shown in Figure 8. Intriguingly, emission spatially associated with filament F1 mainly originates from gas with velocities smaller than $51 \mathrm{~km} \mathrm{~s}^{-1}$ while the gas associated with filaments F2 and F3 is systematically redskewed with velocities larger than $50 \mathrm{~km} \mathrm{~s}^{-1}$. The variation of velocity indicates bulk motions in these filaments.

Spectra of ${ }^{13} \mathrm{CO}(1-0)$ have been extracted along the filaments with a step-size of half the angular resolution $\left(\sim 46^{\prime \prime}\right)$ of the GRS observations, and fitted to a one-dimensional Gaussian profile using the GILDAS/CLASS software. The resultant centroid velocities of the extracted spectra are shown in Figure 9(a) as color-coded circles. The smoothly varying velocity along filaments F1, F2, and F3 is reminiscent of the situation in the SDC13 infrared dark clouds where longitudinal filamentary collapse has been reported (Peretto et al. 2014).

Alternative possible physical processes, which can lead to the observed velocity pattern, include collapse, rotation, filament collision, expansion, and wind-driven acceleration (Peretto et al. 2014). The observed velocity gradients along the filaments in G22 do not allow us to determine a common axis for rotation to take place unless the axis goes through the filament junction. However, such a scenario is unrealistic as differential rotation would tear apart these filaments (Peretto et al. 2014). No signs of enhanced linewidth in the interaction and bridging features (Benjamin et al. 2003; Gong et al. 2017) can be observed in the junction, excluding the possibility of filament collision. No $\mathrm{H}$ II region is observed at the center of $\mathrm{G} 22$. And the outflows driven by MIR1 in clump $\mathrm{C} 1$ cannot explain the red-skewed velocity along F2 as the outflowing gas in that direction is blueshifted (see Figure 5). Similar to the situation in SDC13 (Peretto et al. 2014), there also resides a mid-IR-bright nebula to the southwest of G22 (see Figures 1). This mid-IR nebula corresponds to an infrared bubble MWP1G022027+002159 and is interacting with G22 (see Section 5.4). As MWP1G022027+002159 is located slightly behind G22 (see Section 5.4), its expansion would lead to systematic blueshifted velocities in the interaction region, but the affected area is limited. The largely blue-skewed velocity in the most distant section of F1 and the red-skewed velocity in F2 cannot be explained by wind-driven expansion.

Figure 10(a) shows the difference between the velocities of the filaments and the junction as a function of distance to the center. Monotonically increasing profiles for F1, F2, and F3 are consistent with those observed in other reported collapsing filaments (e.g., SDC13 and AFGL 5142 in Peretto et al. 2014; Liu et al. 2016). The estimated velocity gradients in F1, F2 and $\mathrm{F} 3$ are $0.36,0.35$, and $0.21 \mathrm{~km} \mathrm{~s}^{-1} \mathrm{pc}^{-1}$, which are comparable to those in SDC13 (0.22-0.63, Peretto et al. 2014), and smaller than those in The Serpens South cluster $(\approx 1.4$, Kirk et al. 2013) and AFGL 5142 (9-17, Liu et al. 2016). The small velocity gradients in G22 may indicate low filament inclination.

The velocity dispersion of the filaments as a function of distance to the center is shown in Figure 10(b). For filaments F3 and F4, an increasing trend of velocity dispersion can be seen toward the center region. This increase could be due to additional kinetic energy converted from gravitational energy during the collapse of the filaments (Peretto et al. 2014). Such an increase of velocity dispersion can also be observed toward the inner part of filament F1. In contrast, filament F2 has significantly larger velocity dispersions. As ${ }^{13} \mathrm{CO}(1-0)$ could be optically thick, observations of more optically thin lines, such as $\mathrm{N}_{2} \mathrm{H}^{+}$, would help better reveal the velocity dispersions.

Following Kirk et al. (2013), we estimated the mass infall rates $\dot{M}=\frac{\nabla V M}{\tan (\alpha)}$ to be 132,206 , and $104 M_{\odot} \mathrm{Myr}^{-1}$ in F1, F2, and F3. Here, we assumed an inclination angle of $45^{\circ}$. Therefore, about $440 M_{\odot}$ gas will be channeled to the junction regions in $1 \mathrm{Myr}$ if the current accretion rates are sustained. This high accretion will double the mass of the hub region in about six free-fall times where the free-fall time is about $0.31 \mathrm{Myr}$. Although two million years of high accretion may not be plausible, according to models of the evolution of highmass stars, it is tenable to posit that a fraction of the masses in the central clumps $\mathrm{C} 1$ and and $\mathrm{C} 2$ have been assembled through large-scale filamentary collapse.

\subsection{Clump-fed Accretion in Clump C1}

A virial parameter of $\alpha_{\text {vir }}=5 \sigma_{\text {turb }}^{2} R / G M=0.58$ for clump $\mathrm{C} 1$ has been estimated based on a velocity dispersion of $\sigma_{\text {turb }}=0.87 \mathrm{~km} \mathrm{~s}^{-1}$ measured from the JCMT C ${ }^{18} \mathrm{O}(3-2)$ data. With an $\alpha_{\text {vir }}$ smaller than $1, \mathrm{C} 1$ is likely dominated by 


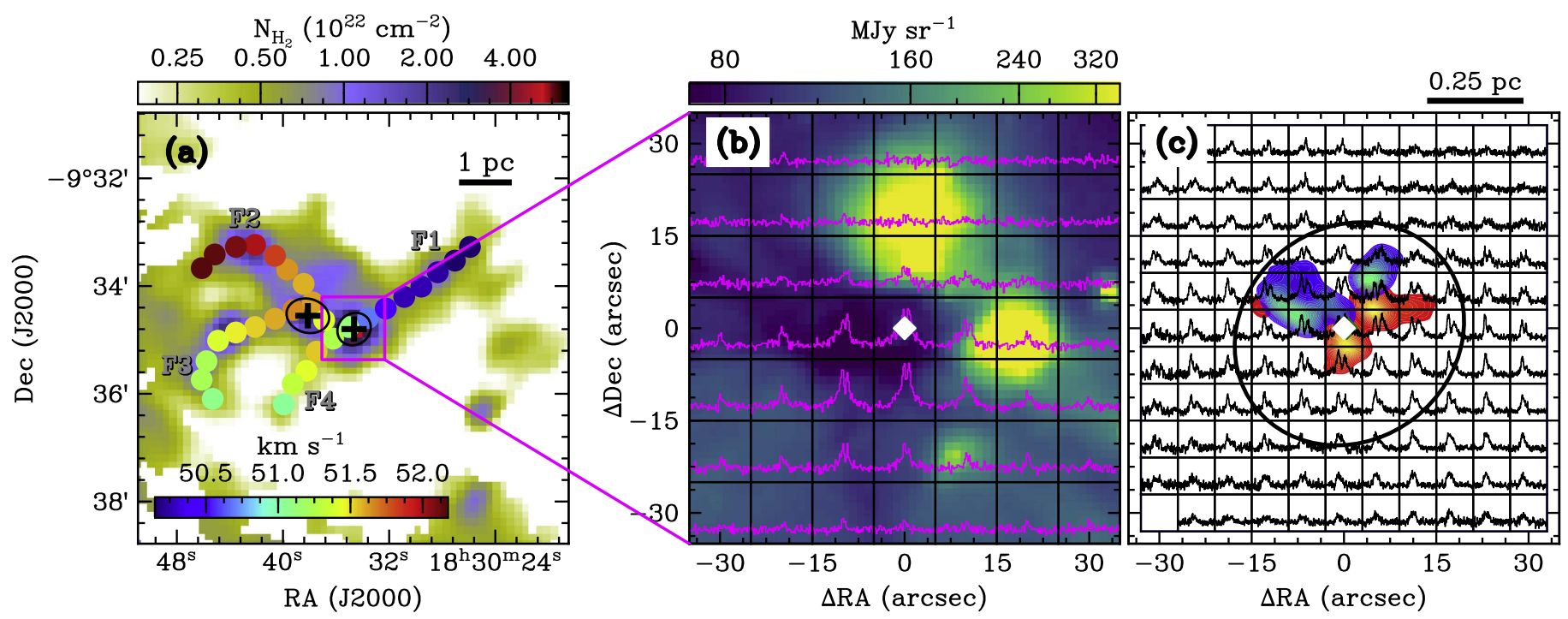

Figure 9. (a) Velocity centroids of ${ }^{13} \mathrm{CO}(1-0)$ extracted along filaments overlaid on top of a $N_{\mathrm{H}_{2}}$ column density map. Also labeled are the two most massive clumps (black "+" and small ellipses) in the hub region. (b) Spectra of JCMT/HCO ${ }^{+}(3-2)$ overlaid on the IRAC $8.0 \mu$ m image. (c) Spectra of JCMT/ ${ }^{13} \mathrm{CO}(3-2)$ overlaid on the SMA/CO (2-1) outflows. The white diamond in panels (b) and (c) labels the $1.3 \mathrm{~mm}$ core SMA1. The large ellipse in (c) delineates clump C1.

gravity and potentially collapsing. Magnetic fields might provide additional support against gravity. Following Pillai et al. (2011), we estimated that the magnetic field strength necessary to virialize $\mathrm{C} 1$ is $B_{\mathrm{vir}}=260 \mu \mathrm{G}$, which is comparable to estimates for the field in clouds at similar densities (Crutcher 2012; Li et al. 2014). Note that the virial parameter may have been overestimated, as any systematic motions, such as infall and outflow, could significantly increase the velocitydispersion estimate.

Figures 9(b) and (c) show JCMT HCO ${ }^{+}(3-2)$ and ${ }^{13} \mathrm{CO}(3-2)$ spectra of clump $\mathrm{C} 1$ overlaid on the IRAC $/ 8.0 \mu \mathrm{m}$ image and SMA CO (2-1) outflows. All spectra with significant emission $(>3 \sigma)$ for both $\mathrm{HCO}^{+}(3-2)$ and ${ }^{13} \mathrm{CO}(3-2)$ show blue-skewed self-absorbed profiles, with the exception of some spectra at the center (for both lines) and the north (for ${ }^{13} \mathrm{CO}(3-2)$ ). Such blue profiles could originate from collapsing or rotating clouds with an inward increasing temperature profile. The different line profiles in the center could be due to the inclusion of emission from the outflow from SMA1. Although we cannot exclude the contribution of rotation, inward motions must occur to explain the dominance of blue profiles in both lines, especially in the clump-confined region (see the large circle in Figure 9(c)). This is further supported by the blue-skewed clump-averaged spectra shown in Figure 11(a). Intriguingly, the absorption dips of the source-averaged $\mathrm{HCO}^{+}$and ${ }^{13} \mathrm{CO}$ spectra are both red-skewed with respect to the systemic velocity. This feature is consistent with a global collapse scenario as suggested in Jin et al. (2016).

We estimated the infall velocity by fitting the clumpaveraged $\mathrm{HCO}^{+}$spectrum using the RATRAN one-dimensional Monte Carlo radiative transfer code (Hogerheijde \& van der Tak 2000). The input parameters include the clump size, density profile, kinematic temperature profile, $\mathrm{HCO}^{+}$fractional abundance $\left(\mathrm{X}_{\mathrm{HCO}^{+}}\right)$, and infall velocity. We deduced a powerlaw density profile $\left(\rho \propto r^{-1.5}\right)$ based on the mass $\left(\sim 590 M_{\odot}\right)$ and radius ( $\sim 0.39 \mathrm{pc})$ of $\mathrm{C} 1$. Following Peretto et al. (2013), we used a constant temperature that is approximately the source-averaged dust temperature of $\mathrm{C} 1$ (21 K, see Table 2). We ran a grid of 880 models with varying $\mathrm{HCO}^{+}$abundance $X_{\mathrm{HCO}^{+}}$in the range [0.6-1.5] $\times 10^{-9}$, infall velocity $v_{\text {in }}$ in the range $[0.05-0.55] \mathrm{km} \mathrm{s}^{-1}$, and velocity dispersion in the range $[0.8-1.5] \mathrm{km} \mathrm{s}^{-1}$. A reduced $\chi^{2}$ parameter was calculated for the averaged spectrum of each model. Note that only the central $4 \mathrm{~km} \mathrm{~s}^{-1}$ portion was considered in calculating reduced $\chi^{2}$ as the emission from the outflows has a large impact on the outer channels of the $\mathrm{HCO}^{+}$spectrum. Fifty-six models with $\chi^{2}-\chi_{\text {best }}^{2}<3$ were considered to be good fits, here $\chi_{\text {best }}^{2}=1.07$. Figure 12 shows the averaged spectra of $\mathrm{HCO}^{+}(3-2)$ of models with $\chi^{2}<3$. From $1 / \chi^{2}$ weighted parameters of all models with good fits, we derived the infall velocity $\left(V_{\text {in }}\right)$, velocity dispersion $\left(\sigma_{\mathrm{v}}\right)$, and $\mathrm{HCO}^{+}$abundance $X_{\mathrm{HCO}^{+}}$to be $V_{\text {in }}=0.31 \pm 0.12 \mathrm{~km} \mathrm{~s}^{-1}, \sigma_{\mathrm{v}}=1.10 \pm 0.05 \mathrm{~km}$ $\mathrm{s}^{-1}$, and $X_{\mathrm{HCO}^{+}}=(1.09 \pm 0.06) \times 10^{-9}$, respectively. We also investigated the affect of varying the radius of the collapse $R_{\text {in }}$ and found that the $\mathrm{HCO}^{+} J=3-2$ can be well modeled only for $R_{\text {in }}>0.5 \mathrm{pc}$. This is consistent with the observed blue-skewed ${ }^{13} \mathrm{CO} J=3-2$ spectra beyond the extent of clump C1 (see Figure 9(c)) and further supports a clump-scale collapse scenario.

We estimated the current mass infall rate using $\dot{M}_{\text {in }}=$ $4 \pi r^{2} \rho v_{\text {in }}=1.5 M v_{\text {in }} / r$ to be about $7.2 \times 10^{-4} M_{\odot} \mathrm{yr}^{-1}$. Here, $\rho \propto r^{-1.5}$ is assumed. The mass infall rate is about five times smaller than the free-fall accretion rate $\left(\dot{M}_{\mathrm{ff}} \sim 3.55 \times\right.$ $10^{-3} M_{\odot} \mathrm{yr}^{-1}$ ) but still significantly larger than the mass outflow rate of SMA1, suggesting that the central hot core can keep growing in mass via clump-fed accretion. Using a velocity dispersion of $0.87 \mathrm{~km} \mathrm{~s}^{-1}$ measured from the JCMT $\mathrm{C}^{18} \mathrm{O}$ (3-2), we estimate the crossing time of $\mathrm{C} 1$ to be $0.43 \mathrm{Myr}$, which is about 2.5 free-fall times $\left(t_{\mathrm{ff}} \sim 0.17 \mathrm{Myr}\right)$. In spite of a difference of about a factor 2 , the crossing and freefall times agree order-of-magnitude wise considering they are just approximate values. This further supports a dynamical global collapse scenario for clump C1.

\subsection{SMA1: A Collapsing Hot Molecular Core}

Most of the 103 molecular lines detected toward the SMA1 peak are from complex organic molecules. This, together with the small size $(0.034 \mathrm{pc})$ and high density $\left(7.2 \times 10^{6} \mathrm{~cm}^{-2}\right)$, 

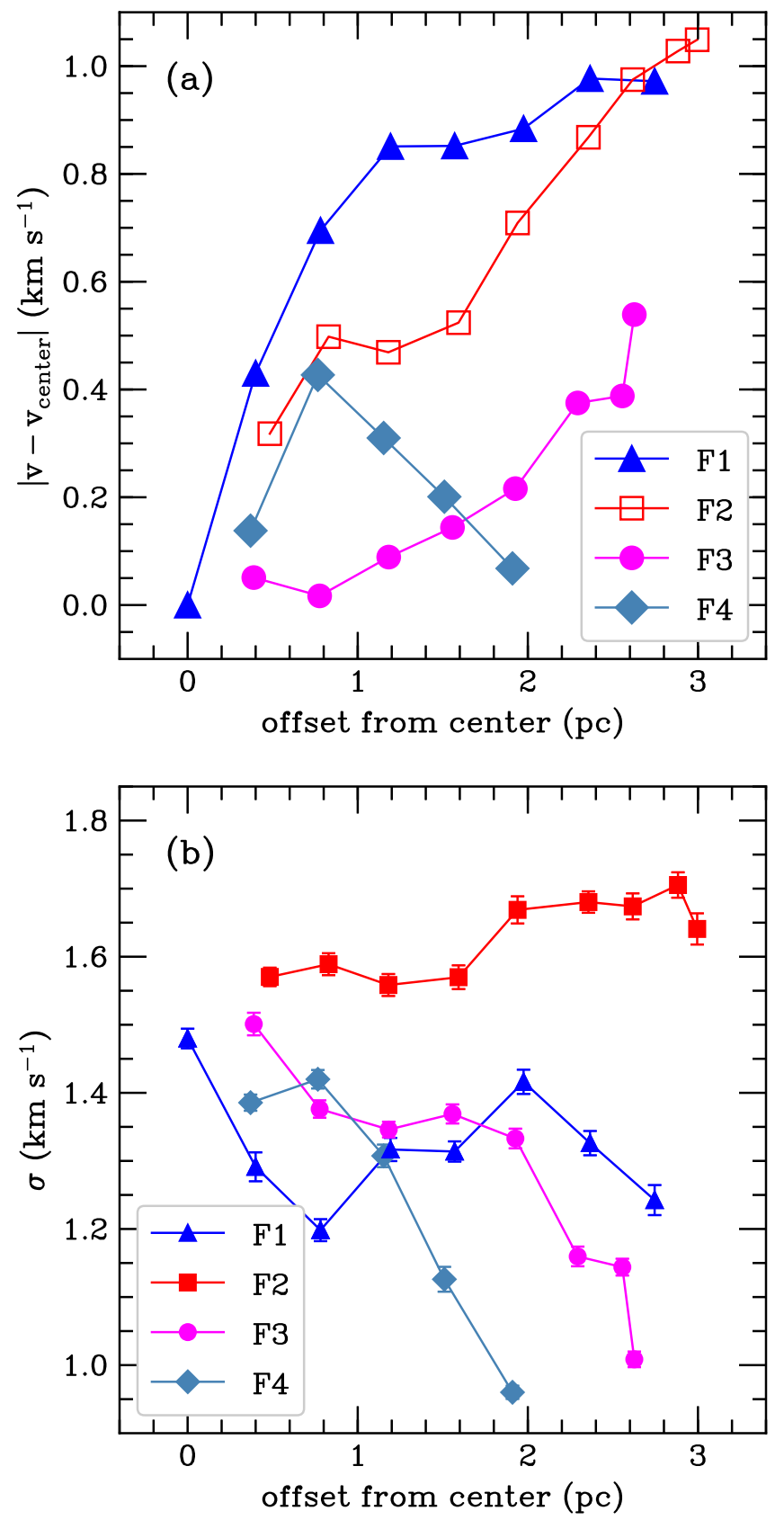

Figure 10. (a) Line-of-sight velocity of ${ }^{13} \mathrm{CO}(1-0)$ as a function of position from the potential well centers, i.e., clump $\mathrm{C} 1$ for $\mathrm{F} 1, \mathrm{~F} 2$, and $\mathrm{F} 4$, and clump $\mathrm{C} 2$ for F3. (b) Velocity dispersion of ${ }^{13} \mathrm{CO}(1-0)$ as a function of offset from the potential well centers.

suggests that SMA1 is a hot molecular core. The physical properties of hot cores are characterized by a small source size $(\leqslant 0.1 \mathrm{pc})$, a high density $\left(\geqslant 10^{6} \mathrm{~cm}^{-2}\right)$, and warm gas/dust temperature ( $\geqslant 100 \mathrm{~K}$; Kurtz et al. 2000; van der Tak 2004).

\subsubsection{High Gas Temperature}

Fifteen $\mathrm{CH}_{3} \mathrm{OH}$ transitions with $E_{\text {up }} / k$ ranging from 40 to $802 \mathrm{~K}$ and eight $\mathrm{CH}_{3} \mathrm{CN}$ transitions with $E_{\text {up }} / k$ ranging from 69 to $419 \mathrm{~K}$ have been reliably detected toward SMA1. Assuming that these lines are optically thin and SMA1 is in LTE, beam-averaged temperatures and column densities of $\mathrm{CH}_{3} \mathrm{OH}$ and $\mathrm{CH}_{3} \mathrm{CN}$ can be obtained using the rotational diagram method (Goldsmith \& Langer 1999). The column
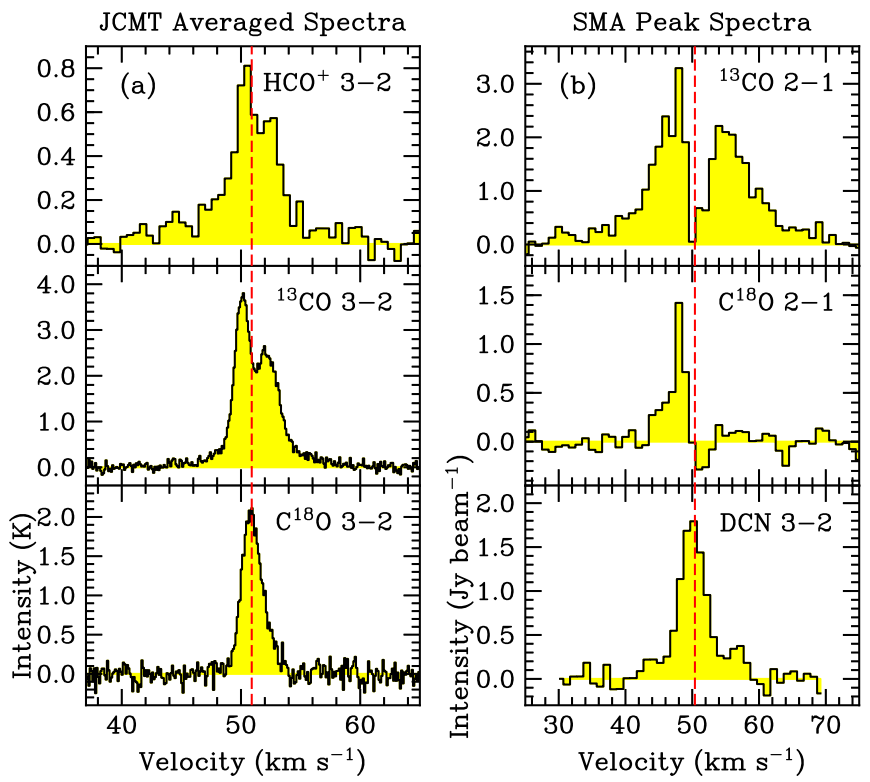

Figure 11. (a) $\mathrm{JCMT} \mathrm{HCO}^{+}(3-2),{ }^{13} \mathrm{CO}(3-2)$, and $\mathrm{C}^{18} \mathrm{O}(3-2)$ spectra averaged over clump $\mathrm{C}$. (b) SMA ${ }^{13} \mathrm{CO}(2-1)$, and $\mathrm{C}^{18} \mathrm{O}(2-1)$ and DCN (3-2) spectra at the peak of SMA1.

density $\left(N_{u}\right)$ of the upper state of a transition can be expressed as a function of its upper state energy $\left(E_{u} / k\right)$,

$$
\ln \frac{N_{u}}{g_{u}}=\ln \frac{N}{Q\left(T_{\mathrm{rot}}\right)}-\frac{E_{u} / k}{T_{\mathrm{rot}}},
$$

where

$$
N_{u}=\frac{8 \pi k \nu^{2}}{h c^{3} A_{u l}} \int T_{b} d v .
$$

Here, $g_{u}$ is the upper state degeneracy, $N$ is the beam-averaged total column density of a species, $Q\left(T_{\text {rot }}\right)$ is the partition function at a given temperature, $T_{\text {rot }}$ is the rotation temperature, $A_{u l}$ is the Einstein A-coefficient for the transition, and $\int T_{b} d v$ is the velocity-integrated intensity of a specific line.

The partition function of $\mathrm{CH}_{3} \mathrm{OH}$ and $\mathrm{CH}_{3} \mathrm{CN}$ can be expressed as,

$$
Q\left(T_{\text {rot }}\right)=a T_{\text {rot }}^{b}
$$

The constants of $a$ and $b$ were obtained by fitting the JPL partition function values at 9.375-300 K.

With multiple transitions observed, which have upper state energies spanning through a large range, the column density and rotation temperature of a specific molecule can be obtained via a least-square fitting to $\ln \frac{N_{u}}{g_{u}}$ as a linear function of $E_{u} / k$.

The fitting results are shown in Figure 13. A rotation temperature of $227 \pm 33 \mathrm{~K}$ and a column density of $(1.01 \pm 0.12) \times 10^{17} \mathrm{~cm}^{-2}$ have been attained for $\mathrm{CH}_{3} \mathrm{OH}$. The resulting temperature and column density for $\mathrm{CH}_{3} \mathrm{CN}$ are $308 \pm 59 \mathrm{~K}$ and $(1.80 \pm 0.26) \times 10^{15} \mathrm{~cm}^{-2}$. The gas temperatures of SMA1 are consistent to those of hot cores in our Galaxy (120-480 K; Hernández-Hernández et al. 2014).

\subsubsection{MIRl: an Embedded High-mass Protostar}

Near the peak of SMA1, there is a mid-IR point source (SSTGLMC G022.0387+0.2222, MIR1 for short). Photometric data collected from the GLIMPSE, MIPSGAL, Hi-GAL, and 


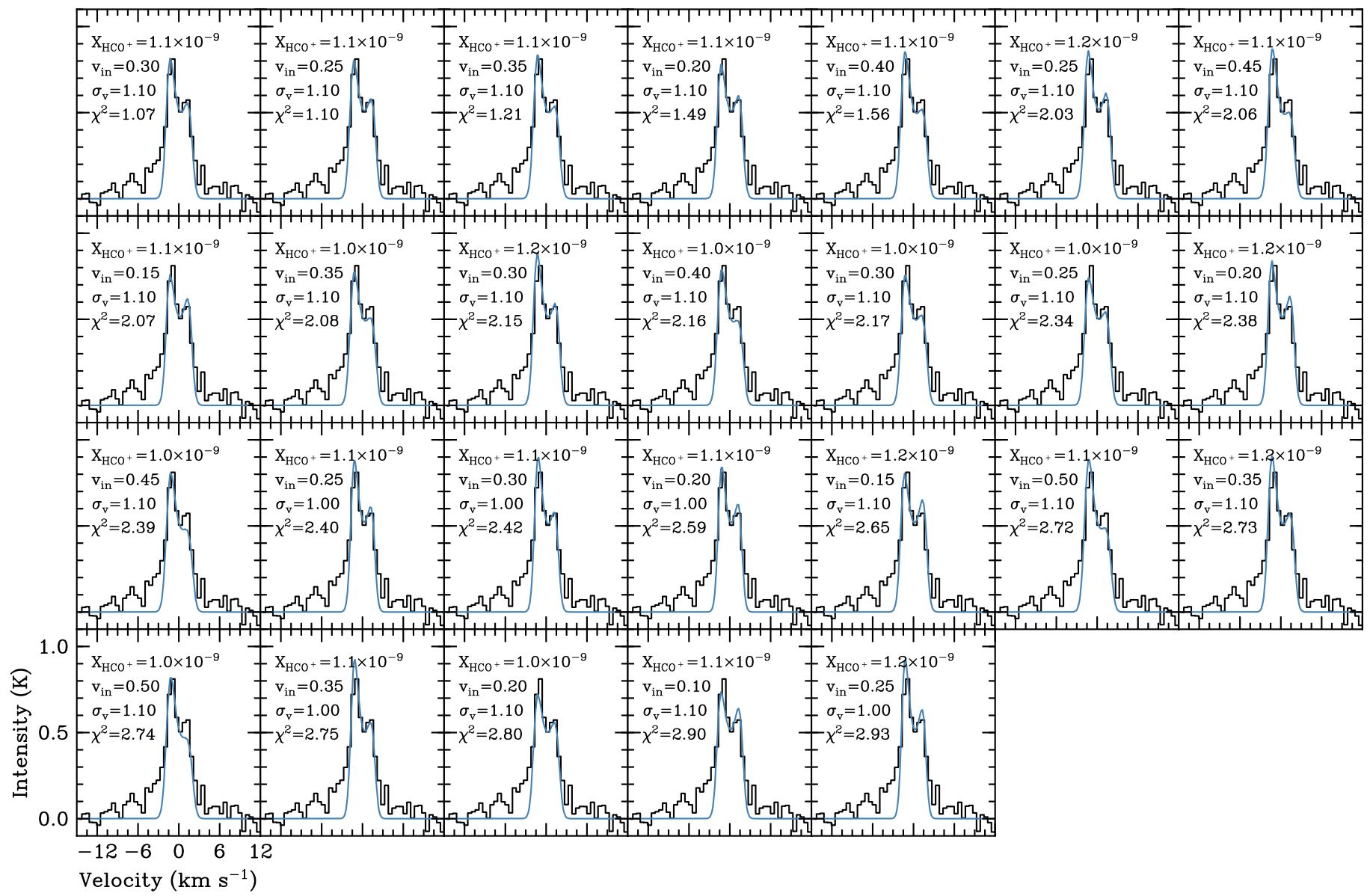

Figure 12. Spectrum of $\mathrm{HCO}^{+}(3-2)$ averaged over clump $\mathrm{C} 1$. The $\mathrm{HCO}^{+}(3-2)$ spectra obtained from RATRAN modeling of a collapsing clump are shown as blue. Details about the modeling are given in Section 5.2. Here, we only show the models with reduced $\chi^{2}$ smaller than 3 . The $\mathrm{HCO}^{+}$abundance, infall velocity, and velocity dispersion are labeled for each model.

ATLASGAL surveys have been fitted to models of YSOs developed by Robitaille et al. (2006, 2007). The resultant SED of MIR1 is shown in Figure 14. The good fits suggest that MIR1 is a Stage $0 / \mathrm{I}$ type high-mass protostar with a stellar mass of [11-15] $M_{\odot}$, a total luminosity of [3.5-7.2] $\times 10^{3} L_{\odot}$, an envelope accretion rate of [1.3-4.1] $\times 10^{-3} M_{\odot} \mathrm{yr}^{-1}$, and an age of $[0.6-3.8] \times 10^{4}$ years.

\subsubsection{Possible Core-fed Accretion}

As shown in Figure 11(b), blue profile and inverse P-cygni profile have been observed in SMA ${ }^{13} \mathrm{CO}(2-1)$ and $\mathrm{C}^{18} \mathrm{O}(2-1)$ spectra. A similar blue-skewed profile is also seen in $\mathrm{CH}_{3} \mathrm{OH}\left(18_{3,16}-17_{4,13} \mathrm{~A}+\right.$ ) (see Figure 15). Although the selfabsorption in these lines can be partially due to the short spacing issues related to interferometer observations, blue-skewed profiles still could be tracing some dynamical motions. Additionally, the inverse P-cygni profile of $\mathrm{C}^{18} \mathrm{O}(3-2)$ cannot be fully explained by short spacing problem, and has been frequently interpreted as a convincing tracer of infall motions in other interferometer observations (Wu et al. 2009; Liu et al. 2011a, 2011b, 2013; Qiu et al. 2011, 2012). And the short spacing problem would filter structures larger than $20^{\prime \prime}$ and cannot significantly alter the profile of $\mathrm{CH}_{3} \mathrm{OH}\left(18_{3,16}-17_{4,13} \mathrm{~A}+\right)$ as this line mainly traces the dense core (see Figure 15(a)). Careful inspection of moment 1 maps of all transitions shows there is no detectable rotation in SMA1 with current SMA observations. This suggests that the blue-skewed profiles could be due to infall motions. Blue profiles of
${ }^{13} \mathrm{CO} J=2-1$ based on SMA observations have also been interpreted as tracing infall in other star-forming regions (e.g., Lee et al. 2006; Wu et al. 2009; Zhang et al. 2014).

Using the model of Myers et al. (1996), an infall velocity of about $0.16 \mathrm{~km} \mathrm{~s}^{-1}$ was estimated. Assuming a density profile of $\rho \propto r^{-1.5}$, we obtain a mass infall rate of $7.0 \times 10^{-5} M_{\odot} \mathrm{yr}^{-1}$, which is a quarter of the free-fall accretion rate $\left(\dot{M}_{\mathrm{ff}} \sim 3 \times\right.$ $10^{-4} M_{\odot} \mathrm{yr}^{-1}$ ) and comparable to mass accretion rates of highmass star-forming cores in numerical studies (Keto 2007; Kuiper et al. 2010, 2011) as well as in interferometric observations (e.g., Liu et al. 2013; Wu et al. 2014). Assuming that about half of the gas flow can be successfully channeled onto the central protostar, MIR1 would grow to an O9 star in about $1 \times 10^{5}$ years if such accretion rate were sustained.

In summary, the gas in G22 is being channeled into the central hub region through global filamentary collapse. The most massive clump $\mathrm{C} 1$ at the center is collapsing and feeding the embedded hot molecular core SMA1. The high-mass protostar MIR1 in SMA1 is also gaining mass from SMA1 where infall motions have been tentatively detected.

\subsection{Influence from the Adjacent Bubble}

The infrared bubble MWP1G022027+0022159 has been observed in the south of G22 (see Figures 1 and 2(b)). The dark lane observed to the near-IR suggests the bubble is behind G22. This is also supported by the changes of velocities from red to blue for masers associated with the outflow lobe R1 (Figure 5). 

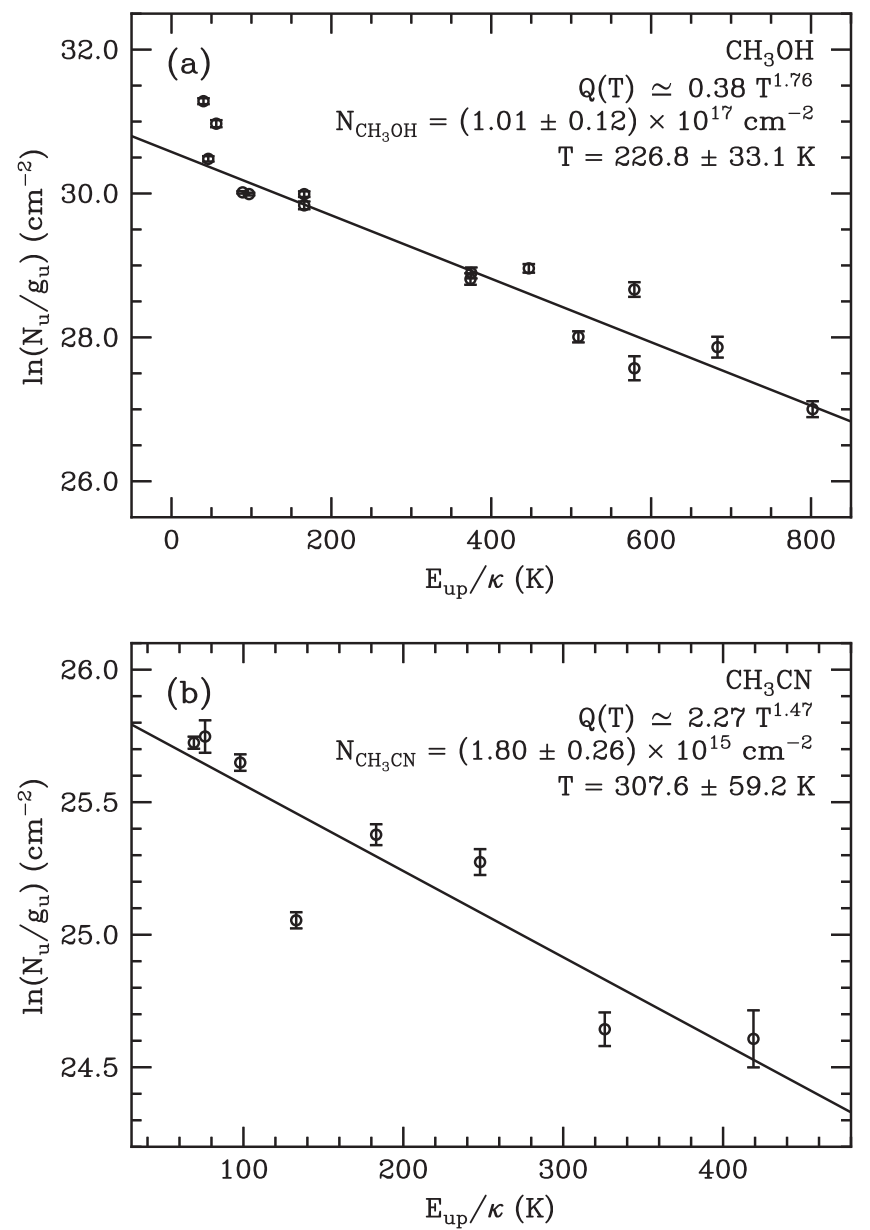

Figure 13. Rotation diagrams of $\mathrm{CH}_{3} \mathrm{OH}$ (a) and $\mathrm{CH}_{3} \mathrm{CN}$ (b).

The reversal of maser velocities and large intensity gradients of R1 suggest that the bubble might be strongly interacting with G22 and modifying the direction of the outflowing gas.

As the bubble is slightly behind G22, its expansion is apt to push the gas in G22 toward us. And the interacting region would have a higher temperature and more blueshifted velocities. Thus, any existing self-absorption due to the bubble would result in line profiles with a higher red peak (i.e., red profiles). The ubiquitous blue profiles in $\mathrm{C} 1$ cannot originate from the the influence of the IR bubble. However, an expansion of the bubble would introduce a blue wing to the spectrum of $\mathrm{HCO}^{+} J=3-2$ as shown in Figures 11 and 12 .

\section{Summary}

We have presented a detailed study of a hub-filament system in the G22 cloud. The main findings of this work are summarized as follows:

1. The G22 cloud is composed of four supercritical filaments with mass per unit length ranging from 54 to $220 M_{\odot} \mathrm{pc}^{-1}$. Velocity gradients along three filaments revealed by ${ }^{13} \mathrm{CO} J=1-0$ indicates they are collapsing and channeling gas toward the junction. A total mass infall rate of $440 M_{\odot} \mathrm{Myr}^{-1}$ suggests that the hub mass would be doubled in six free-fall times. Although such a high accretion may not be sustained for almost two million years, it is tenable to posit that a fraction of the

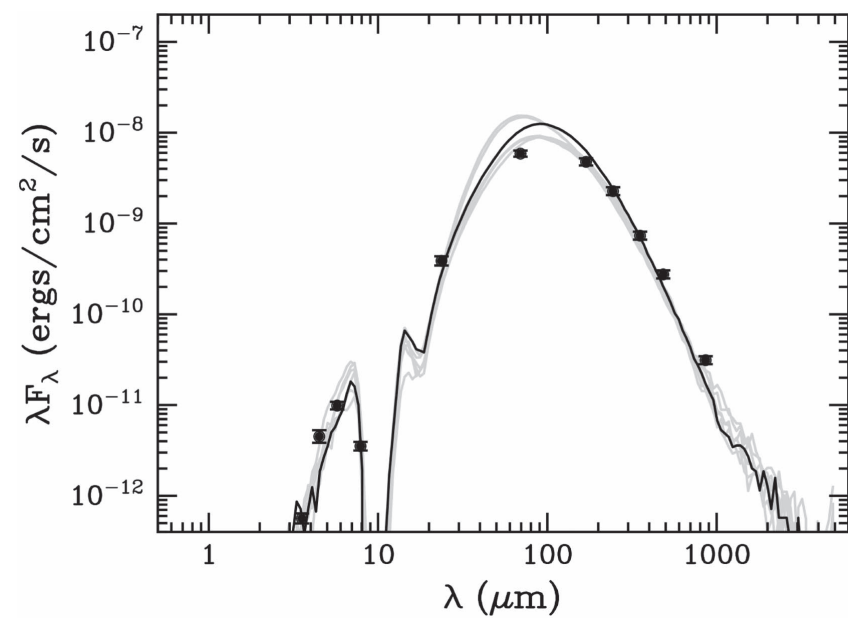

Figure 14. Spectral energy distribution of MIR1 (SSTGLMC G022.0387 +00.2222 ). Photometric data from GLIMPSE, MIPSGAL, Hi-GAL, and ATLASGAL were fitted using YSO models of Robitaille et al. (2006, 2007). The black line shows the best fit, and the gray lines show subsequent good fits with $\left(\chi^{2}-\chi_{\text {best }}^{2}\right)<3 N$.
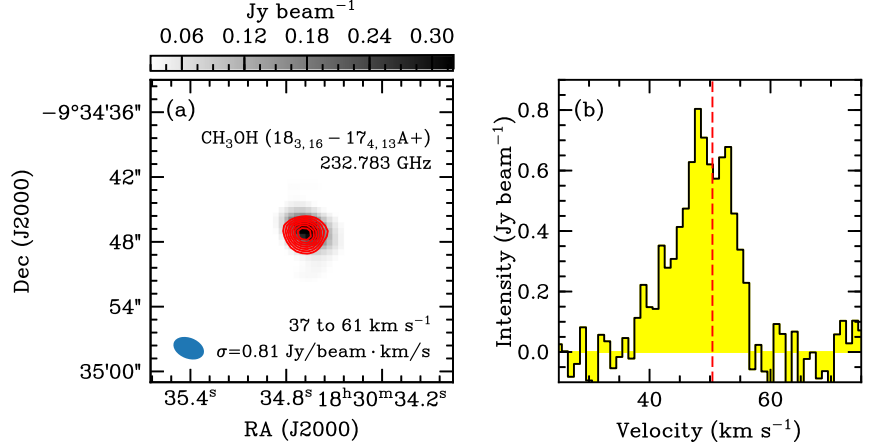

Figure 15. (a) Velocity integrated intensity of $\mathrm{CH}_{3} \mathrm{OH}\left(18_{3,16}-17_{4,13} \mathrm{~A}+\right.$ ) overlaid on the $1.3 \mathrm{~mm}$ continuum image. Contours start from $3 \sigma$ with an increasing step of $1 \sigma$. (b) Spectrum of $\mathrm{CH}_{3} \mathrm{OH}\left(18_{3,16}-17_{4,13} \mathrm{~A}+\right)$ at the SMA1 peak. The vertical dashed line marks the systemic velocity $\left(50.4 \mathrm{~km} \mathrm{~s}^{-1}\right)$ of SMA1.

mass in the central clumps $\mathrm{C} 1$ and $\mathrm{C} 2$ have been built through large-scale filamentary collapse.

2. The most massive and densest clump $\mathrm{C} 1$ at the junction is globally collapsing. With a virial parameter $\alpha_{\text {vir }}<1, \mathrm{C} 1$ is supercritical unless strong magnetic fields can provide additional support. Prevalent blue profiles of $\mathrm{HCO}^{+}(3-2)$ and ${ }^{13} \mathrm{CO}(3-2)$ spectra support a scenario of clump-scale collapse. An infall velocity of $0.31 \mathrm{~km} \mathrm{~s}^{-1}$ was estimated via modeling the clump-averaged $\mathrm{HCO}^{+}$spectrum using the RATRAN code. The inferred mass infall rate is about $7.2 \times 10^{-4} M_{\odot} \mathrm{yr}^{-1}$.

3. Embedded in clump C1, a hot molecular core (SMA1) has been revealed by the SMA observations. More than 100 lines from 19 species (26 isotopologues including complex organic molecules) have been detected. The gas temperature was estimated to be higher than $200 \mathrm{~K}$ via fitting the rotation diagrams of $\mathrm{CH}_{3} \mathrm{OH}$ and $\mathrm{CH}_{3} \mathrm{CN}$. The detected outflows and the Class-I-like SED of the embedded mid-infrared source suggest that high-mass star formation is taking place in SMA1. The high-mass star-forming hot core SMA1 may be still growing in mass via clump-fed accretion. This is supported by the 
high-mass infall rate of $\mathrm{C} 1$, which is significantly larger than the mass loss rate of the outflows.

4. A high-mass protostar (MIR1) is located at the center of SMA1 and could be the driving source of the observed outflows. The mass of MIR1 is estimated to be in the range [11-15] $M_{\odot}$ and may be still growing via core-fed accretion, which is supported by the detected infall features in SMA1.

5. Anomalous methanol emission of $\mathrm{CH}_{3} \mathrm{OH}\left(8_{-1,8}-7_{0,7}\right)$ at $229.759 \mathrm{GHz}$ and $\mathrm{CH}_{3} \mathrm{OH}\left(4_{2,2}-3_{1,2}\right)$ at $218.440 \mathrm{GHz}$ was detected in three positions around SMA1. Large $8_{-1,8^{-}} 7_{0,7} / 3_{-2,2}-4_{-1,4}$ and $4_{2,2}-3_{1,2} / 3_{-2,2}-4_{-1,4}$ ratios suggest maser emission of these two methanol transitions.

The coexistence of infall through filaments, clumps, and the central core has revealed continuous mass growth from large to small scales. This suggests that pre-assembled mass reservoirs may not be indispensable to form high-mass stars. In the process of high-mass star formation, the masses of the central protostar, the core, and the clump can simultaneously grow via core-fed/disk accretion, clump-fed accretion, and filamentary/global collapse.
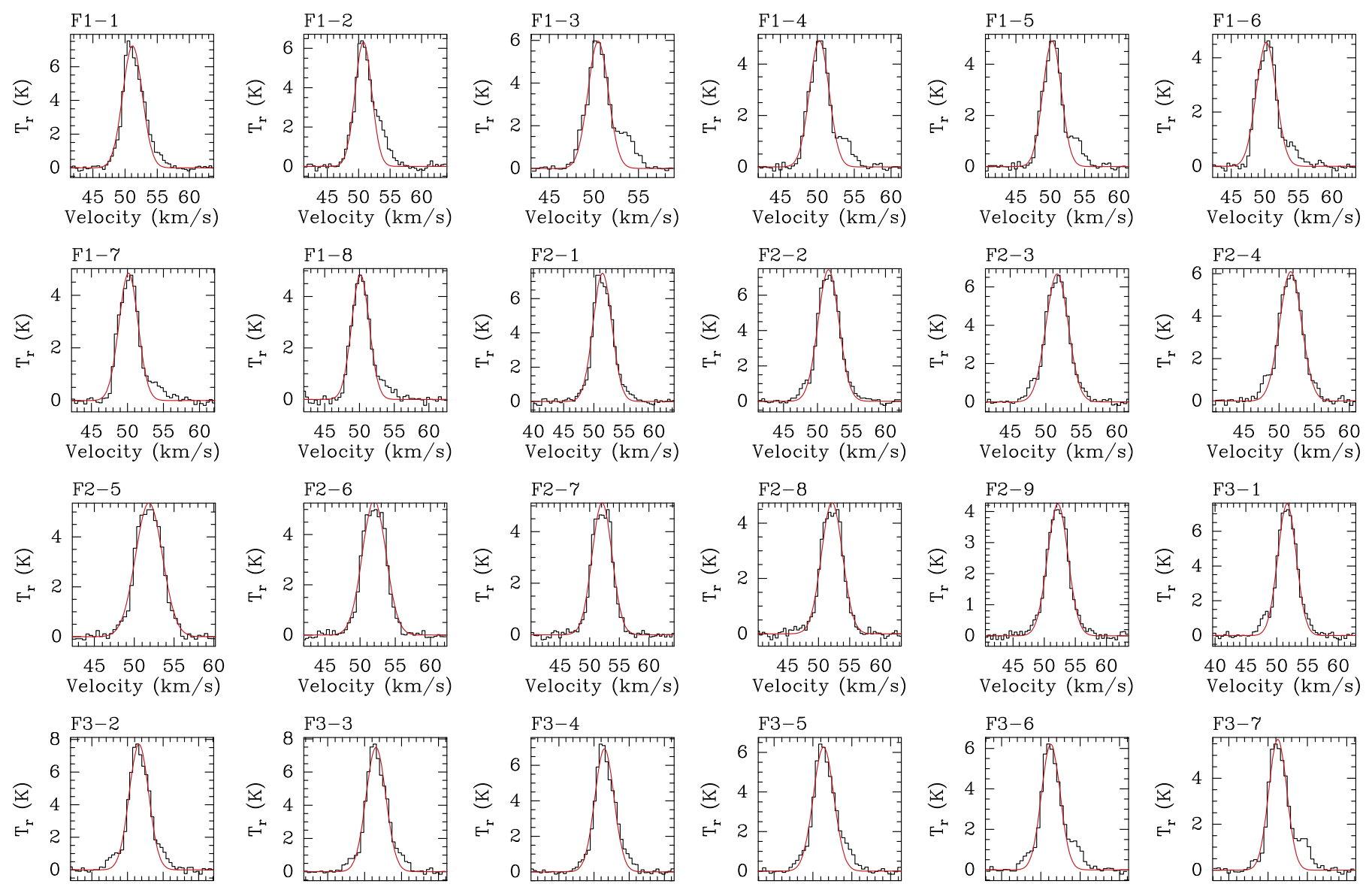

$45 \quad 50 \quad 55 \quad 60$ Velocity $(\mathrm{km} / \mathrm{s})$
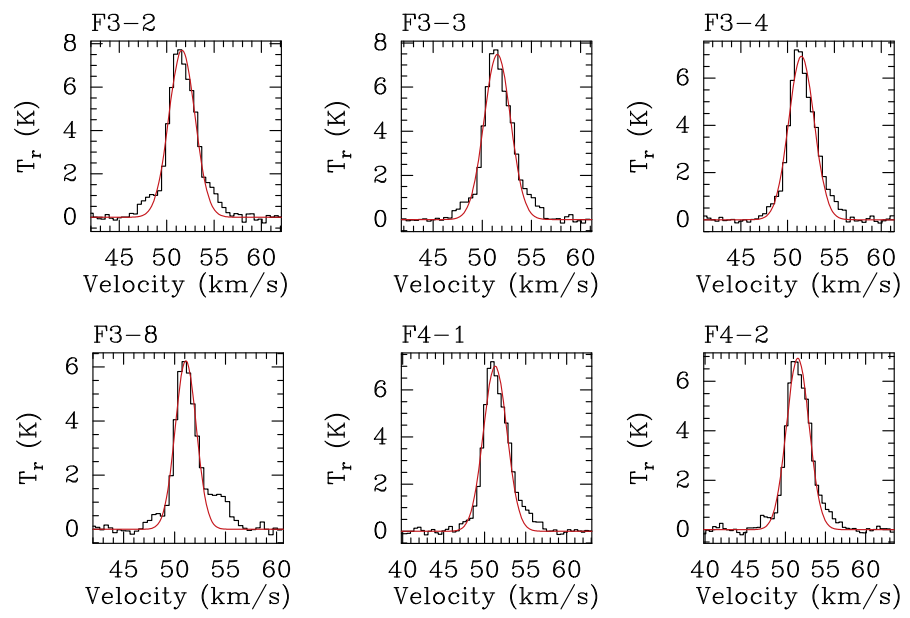
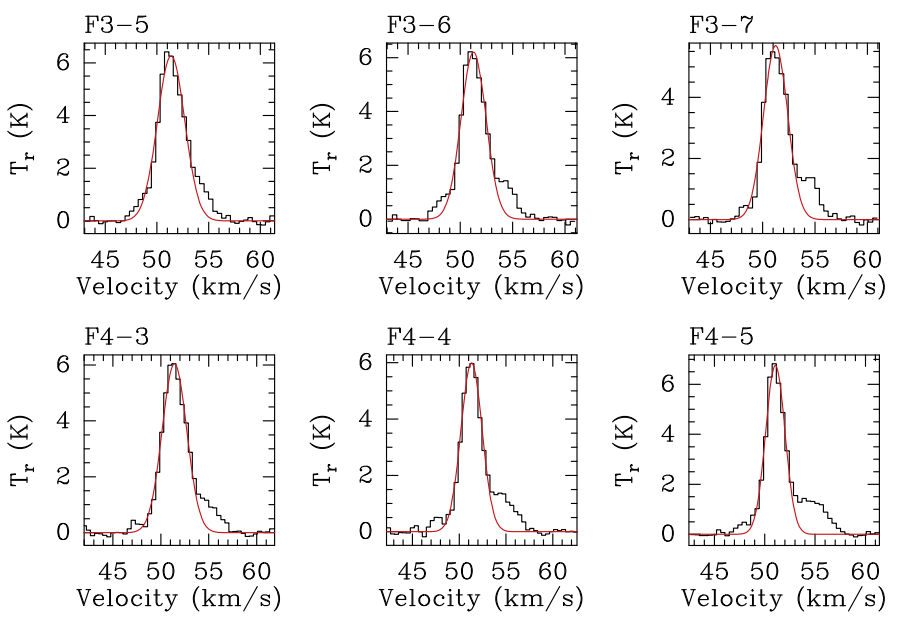

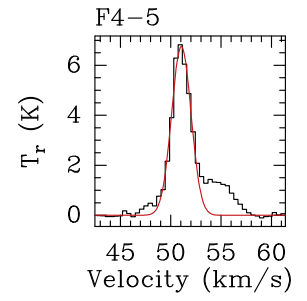

Figure 16. Spectra of ${ }^{13} \mathrm{CO} 1-0$ along filaments. The red line in each panel shows the one-dimensional Gaussian fitting result. 


\section{ORCID iDs}

Jinghua Yuan (iD https://orcid.org/0000-0001-8060-3538

Yuefang Wu (i) https://orcid.org/0000-0002-5076-7520

Simon P. Ellingsen (10 https://orcid.org/0000-0002-1363-5457

Christian Henkel ib https://orcid.org/0000-0002-7495-4005

Ke Wang (i) https://orcid.org/0000-0002-7237-3856

Tie Liu (1) https://orcid.org/0000-0002-5286-2564

Hong-Li Liu (iD https://orcid.org/0000-0003-3343-9645

\section{References}

André, P., Di Francesco, J., Ward-Thompson, D., et al. 2014, in Protostars and Planets VI, ed. H. Beuther et al. (Tucson, AZ: Univ. Arizona Press), 27

André, P., Men'shchikov, A., Bontemps, S., et al. 2010, A\&A, 518, L102

Aniano, G., Draine, B. T., Gordon, K. D., \& Sandstrom, K. 2011, PASP, 123,1218

Astropy Collaboration, Robitaille, T. P., Tollerud, E. J., et al. 2013, A\&A, 558, A33

Beltrán, M. T., Cesaroni, R., Zhang, Q., et al. 2011, A\&A, 532, A91

Benjamin, R. A., Churchwell, E., Babler, B. L., et al. 2003, PASP, 115, 953

Bonnell, I. A., Bate, M. R., Clarke, C. J., \& Pringle, J. E. 2001, MNRAS, 323,785

Chen, X., Ellingsen, S. P., Shen, Z.-Q., Titmarsh, A., \& Gan, C.-G. 2011, ApJS, 196, 9

Churchwell, E., Babler, B. L., Meade, M. R., et al. 2009, PASP, 121, 213

Crutcher, R. M. 2012, ARA\&A, 50, 29

Csengeri, T., Bontemps, S., Schneider, N., Motte, F., \& Dib, S. 2011, A\&A, 527, A135

Cyganowski, C. J., Brogan, C. L., Hunter, T. R., et al. 2012, ApJL, 760, L20

Cyganowski, C. J., Brogan, C. L., Hunter, T. R., \& Churchwell, E. 2009, ApJ, 702,1615

Cyganowski, C. J., Brogan, C. L., Hunter, T. R., Churchwell, E., \& Zhang, Q. 2011, ApJ, 729, 124

Cyganowski, C. J., Whitney, B. A., Holden, E., et al. 2008, AJ, 136, 2391

Federrath, C., \& Klessen, R. S. 2013, ApJ, 763, 51

Federrath, C., Klessen, R. S., \& Schmidt, W. 2008, ApJL, 688, L79

Fontani, F., Zhang, Q., Caselli, P., \& Bourke, T. L. 2009, A\&A, 499, 233

Girichidis, P., Konstandin, L., Whitworth, A. P., \& Klessen, R. S. 2014, ApJ, 781,91

Goldsmith, P. F., \& Langer, W. D. 1999, ApJ, 517, 209

Gong, Y., Fang, M., Mao, R., et al. 2017, ApJL, 835, L14

Hennemann, M., Motte, F., Schneider, N., et al. 2012, A\&A, 543, L3

Hernández-Hernández, V., Zapata, L., Kurtz, S., \& Garay, G. 2014, ApJ, 786,38

Hogerheijde, M. R., \& van der Tak, F. F. S. 2000, A\&A, 362, 697

Hunter, T. R., Brogan, C. L., Cyganowski, C. J., \& Young, K. H. 2014, ApJ, 788,187

Inutsuka, S.-I., \& Miyama, S. M. 1997, ApJ, 480, 681

Ioannidis, G., \& Froebrich, D. 2012, MNRAS, 421, 3257

Jackson, J. M., Rathborne, J. M., Shah, R. Y., et al. 2006, ApJS, 163, 145

Jin, M., Lee, J.-E., Kim, K.-T., \& Evans, N. J., II 2016, ApJS, 225, 21

Kauffmann, J., Bertoldi, F., Bourke, T. L., Evans, N. J., II, \& Lee, C. W. 2008, A\&A, 487, 993

Keto, E. 2007, ApJ, 666, 976

Kirk, H., Myers, P. C., Bourke, T. L., et al. 2013, ApJ, 766, 115

Klessen, R. S., \& Hennebelle, P. 2010, A\&A, 520, A17

Kritsuk, A. G., Norman, M. L., \& Wagner, R. 2011, ApJL, 727, L20

Kuiper, R., Klahr, H., Beuther, H., \& Henning, T. 2010, ApJ, 722, 1556

Kuiper, R., Klahr, H., Beuther, H., \& Henning, T. 2011, ApJ, 732, 20

Kurtz, S., Cesaroni, R., Churchwell, E., Hofner, P., \& Walmsley, C. M. 2000, in Protostars and Planets IV, ed. V. Manning et al. (Tucson, AZ: Univ. Arizona Press), 299

Lee, C.-F., Ho, P. T. P., Beuther, H., et al. 2006, ApJ, 639, 292

Li, H.-B., Goodman, A., Sridharan, T. K., et al. 2014, in Protostars and Planets VI, ed. H. Beuther et al. (Tucson, AZ: Univ. Arizona Press), 101

Liu, H. B., Quintana-Lacaci, G., Wang, K., et al. 2012, ApJ, 745, 61
Liu, T., Lacy, J., Li, P. S., et al. 2017, arXiv:1705.04907

Liu, T., Wu, Y., Liu, S.-Y., et al. 2011a, ApJ, 730, 102

Liu, T., Wu, Y., Wu, J., Qin, S.-L., \& Zhang, H. 2013, MNRAS, 436, 1335

Liu, T., Wu, Y., Zhang, Q., et al. 2011b, ApJ, 728, 91

Liu, T., Zhang, Q., Kim, K.-T., et al. 2016, ApJ, 824, 31

McDowell, R. S. 1988, JChPh, 88, 356

McKee, C. F., \& Tan, J. C. 2003, ApJ, 585, 850

Molinari, S., Swinyard, B., Bally, J., et al. 2010a, A\&A, 518, L100

Molinari, S., Swinyard, B., Bally, J., et al. 2010b, PASP, 122, 314

Motte, F., Bontemps, S., \& Louvet, F. 2017, arXiv:1706.00118

Myers, P. C., Mardones, D., Tafalla, M., Williams, J. P., \& Wilner, D. J. 1996, ApJL, 465, L133

Ossenkopf, V., \& Henning, T. 1994, A\&A, 291, 943

Ostriker, J. 1964, ApJ, 140, 1056

Peretto, N., \& Fuller, G. A. 2009, A\&A, 505, 405

Peretto, N., Fuller, G. A., André, P., et al. 2014, A\&A, 561, A83

Peretto, N., Fuller, G. A., Duarte-Cabral, A., et al. 2013, A\&A, 555, A112

Pety, J. 2005, in SF2A-2005: Semaine de l'Astrophysique Francaise, ed. F. Casoli et al. (Strasbourg: Edp-Sciences), 721

Pillai, T., Kauffmann, J., Wyrowski, F., et al. 2011, A\&A, 530, A118

Pineda, J. L., Langer, W. D., Velusamy, T., \& Goldsmith, P. F. 2013, A\&A, 554, A103

Planck Collaboration, Ade, P. A. R., Aghanim, N., et al. 2014, A\&A, 564, A45

Qiu, K., \& Zhang, Q. 2009, ApJL, 702, L66

Qiu, K., Zhang, Q., Beuther, H., \& Fallscheer, C. 2012, ApJ, 756, 170

Qiu, K., Zhang, Q., \& Menten, K. M. 2011, ApJ, 728, 6

Qiu, K., Zhang, Q., Wu, J., \& Chen, H.-R. 2009, ApJ, 696, 66

Reid, M. J., Dame, T. M., Menten, K. M., \& Brunthaler, A. 2016, ApJ, 823, 77

Ren, Z., Wu, Y., Zhu, M., et al. 2012, MNRAS, 422, 1098

Robitaille, T. P., Meade, M. R., Babler, B. L., et al. 2008, AJ, 136, 2413

Robitaille, T. P., Whitney, B. A., Indebetouw, R., \& Wood, K. 2007, ApJS, 169,328

Robitaille, T. P., Whitney, B. A., Indebetouw, R., Wood, K., \& Denzmore, P. 2006, ApJS, 167, 256

Rygl, K. L. J., Wyrowski, F., Schuller, F., \& Menten, K. M. 2013, A\&A, 549, A5

Sault, R. J., Teuben, P. J., \& Wright, M. C. H. 1995, in ASP Conf. Ser. 77, Astronomical Data Analysis Software and Systems IV, ed. R. A. Shaw, H. E. Payne, \& J. J. E. Hayes (San Francisco, CA: ASP), 433

Schneider, N., Csengeri, T., Bontemps, S., et al. 2010, A\&A, 520, A49

Schneider, N., Csengeri, T., Klessen, R. S., et al. 2015a, A\&A, 578, A29

Schneider, N., Ossenkopf, V., Csengeri, T., et al. 2015b, A\&A, 575, A79

Schuller, F., Menten, K. M., Contreras, Y., et al. 2009, A\&A, 504, 415

Simpson, R. J., Povich, M. S., Kendrew, S., et al. 2012, MNRAS, 424, 2442

Slysh, V. I., Kalenskiı̌, S. V., \& Val'tts, I. E. 2002, ARep, 46, 49

Tan, J. C., Beltrán, M. T., Caselli, P., et al. 2014, in Protostars and Planets VI, ed. H. Beuther et al. (Tucson, AZ: Univ. Arizona Press), 149

Tigé, J., Motte, F., Russeil, D., et al. 2017, arXiv:1703.09839

van der Tak, F. F. S. 2004, in IAU Symp. 221, Star Formation at High Angular Resolution, ed. M. G. Burton, R. Jayawardhana, \& T. L. Bourke (Cambridge: Cambridge Univ. Press), 59

Voronkov, M. A., Caswell, J. L., Ellingsen, S. P., et al. 2012, in IAU Symp. 287, ed. R. S. Booth, W. H. T. Vlemmings, \& E. M. L. Humphreys (Cambridge: Cambridge Univ. Press), 433

Wang, K., Testi, L., Burkert, A., et al. 2016, ApJS, 226, 9

Wang, K., Testi, L., Ginsburg, A., et al. 2015, MNRAS, 450, 4043

Wang, K., Zhang, Q., Testi, L., et al. 2014, MNRAS, 439, 3275

Wang, K., Zhang, Q., Wu, Y., \& Zhang, H. 2011, ApJ, 735, 64

Werner, M. W., Roellig, T. L., Low, F. J., et al. 2004, ApJS, 154, 1

Wu, Y., Liu, T., \& Qin, S.-L. 2014, ApJ, 791, 123

Wu, Y., Qin, S.-L., Guan, X., et al. 2009, ApJL, 697, L116

Yuan, J., Wu, Y., Ellingsen, S. P., et al. 2017, ApJS, 231, 11

Zapata, L. A., Loinard, L., Su, Y.-N., et al. 2012, ApJ, 744, 86

Zhang, C.-P., Wang, J.-J., Xu, J.-L., Wyrowski, F., \& Menten, K. M. 2014, ApJ, 784, 107 